\title{
LA CROISSANCE ÉCONOMIQUE FRANÇAISE
}

par des membres de l'Institut de Science Economique Appliquée

I. INTRODUCTION PAR LE PROFESSEUR FRANÇOIS PERROUX

LE document qu'on va lire, présenté par 1'T.S.E.A. à la réunion de 1951 de l'I.A.R.I.W., comporte deux parties: le rapport proprement dit, qui rassemble des chiffres globaux plus ou moins représentatifs du revenu national français pour un certain nombre d'années depuis 1780 , et une annexe qui établit des comptabilités nationales sommaires à trois dates éloignées.

Cette présentation a été choisie par souci de fidélité à ce qui nous était demandé. Livrés à nous-mêmes, nous eussions présenté une série de structures dans le rapport fondamental et renvoyé en annexe le relevé des revenus globaux: la croissance n'a de signification pour nous que comme phénomène de transformation des structures dont il ne peut-être rendu compte, comme le dit G. Th. Guilbaud, par '1'histoire d'un chiffre unique'. J'ai eu maintes occasions, dans des articles et des cours, de manifester mes propres positions sur ce point; il m'a été précieux de me trouver en accord avec mes amis de l'I.S.E.A.

Je remercie très vivement les auteurs des textes qui suivent:

- Jacques Mayer, dont la 'Note sur la structure de 1'Économie française à trois époques éloignées' constitue une très importante contribution au travail de notre équipe et représente une remarquable et scrupuleuse analyse des documents originaux;

- Jean Albert et Marcel Malissen, qui ont procédé avec beaucoup d'intelligence et de persévérance à la recherche et à l'interprétation des sources;

- G. Th. Guilbaud, qui a guidé Albert et Malissen dans leurs recherches et a mis en œuvre les résultats de celles-ci en établissant 1' 'État provisoire des recherches' sur 'Le revenu national français de 1780 à nos jours'.

Je m'associe pleinement aux réserves de Guilbaud sur la signification de la tâche ingrate qu'il a bien voulu assumer et à son espoir de présenter un jour un véritable tableau de la croissance économique française sous la forme d'une histoire raisonnée 'du système de chiffres que constitue une comptabilité nationale'.

Françols Perroux 
II. LE REVENU NATIONAL FRANÇAIS DE 1780 À NOS JOURS

1. La plupart des chercheurs qui ont eu besoin de connaître le revenu national français à diverses époques ont déclaré que la documentation est relativement abondante. C'est ce que dit A. de Foville, ${ }^{1}$ c'est ce que répète Colin Clark dans The Conditions of Economic Progress, ${ }^{2}$ 'for the period between 1789 and 1911 , it is literally true to say that estimates of national income in France were as numerous as for the rest of Europe'. Et de fait il ne serait pas difficile d'établir une liste très longue même en excluant les simples répétitions: pour le cours du 19ème siècle Foville donne 15 estimations, Robert Meyer en donne 10, Clark une trentaine; quant à nous, après examen et sélection, nous en présenterons une cinquantaine, à peu près uniformément réparties dans le temps.

Mais à collectionner les évaluations, on risque fort de voir se constituer une sorte de Vulgate qui se transmettra de texte en texte, mème lorsque les auteurs accompagnent leur tableau de chasse de réserves importantes sur la signification douteuse et 1 'hétérogénéité certaine d'un grand nombre de sources. Notre premier dessein a donc été de remonter à l'origine pour chaque chiffre allégué, surtout pour les chiffres souvent recopiés, de dresser un tableau (dont ou trouvera ci-après la forme provisoire) présentant les sources, les estimations et leur portée. Mais ce tableau, même très critique, ne pouvait être notre but: c'était plutôt un sous-produit de notre travail de recherches. Pour nous, la collecte des chiffres globaux du revenu national annuel en France depuis 1780, et l'étude subséquente de l'évolution de ce revenu, ne présentent qu'un intérêt mineur: ce ne peut être qu'une introduction à l'histoire, non d'un chiffre unique, mais du système de chiffres que constitue une comptabilité nationale. Notre objectif fondamental est, en effet, de rassembler les matériaux nécessaires à une étude de la croissance de l'économie française, dont nous pensons qu'elle ne peut se faire si les chiffres ne sont pas suffisamment décomposés et si, pour le dire de façon brève, nous n'arrivons pas à éclairer le 'financement' de la croissance. Cependant, comme nous l'avions espéré, la prospection préliminaire des sources nous a prouvé que 1'éta-

${ }^{1}$ A. de Foville, La Richesse de la France et la France Economique, 1890, p. 506.

2 Colin Clark, The Conditions of Economic Progress, Macmillan, 1st edition, 1940, p. 99; 2nd edition, 1951, p. 71. 
blissement de comptabilités nationales échelonnées à diverses dates depuis 1780 est loin d'être impossible.

2. Il existe de nombreuses listes groupant des estimations de diverses provenances (Foville, Vignes, Neumann-Spallart, Robert Meyer, Simiand, Colin Clark, etc.), mais aucune ne peut être directement utilisée faute d'avoir associé aux chiffres des commentaires suffisants. D'autre part, à force de recopies mutuelles et parfois insouciantes, les doublets deviennent inévitables; ainsi on pourrait s'émerveiller de quelques beaux accords tels que:

\section{Neumann-Spallart 25 milliards} 1893. Foville 25 milliards

que nous trouvons chez Clark. Mais les Uebersichten du premier auteur se contentent de citer le second dont, par contre, l'estimation est originale et, bien que sommaire, présentée avec justifications. D'une façon générale, la méfiance doit être de règle chaque fois que 1'on trouve deux chiffres identiques: dans la liste de Clark, la règle est efficace à quatre reprises. Le danger d'utiliser des listes toutes faites et d'attribuer la responsabilité des chiffres à un auteur qui n'a fait que glaner (bien souvent au hasard) apparait encore plus grand quand on constate, par exemple, que Colin Clark écrit: 'Of the various discrepant series included in the previous table, Neumann-Spallart's have at least the merit of having been prepared by a single author, and probably were to some extent reckoned on a comparable basis. They should, therefore, be given more consideration than the others,' 1 et quand, se reportant à 1'ouvrage de Spallart ${ }^{2}$ on s'aperçoit que celui-ci nous donne seulement un recueil d'évaluations faites par divers auteurs et qu'il emprunte lui-mème à Robert Meyer et à Foville. La liste de Meyer, qu'on trouve dans le Handwôrterbuch de Conrad est d'ailleurs plus étendue que celle de Spallart mais elle n'est pas non plus originale: sa source principale est l'ouvrage de Schnitzler;' ${ }^{3}$ la liste de Schnitzler est prolongée après 1848 par diverses évaluations postérieures et Meyer signale pour certaines qu'il s'agit d'estimations faites en passant et sans grande valeur statistique. Cette prudence, qui se perdra avec les recopies, est tout à fait justifiée. Ainsi l'une

${ }^{1}$ Clark, op. cit., 1 st edition, p. 103; 2nd edition, p. 74.

2 Neumann-Spallart, Uebersichten der Weltwirtschaft.

${ }^{3}$ Schnitzler, De la Creation de la Richesse, Paris, 1842. 
des sources, qui figure finalement dans le Tableau de Clark sous l'autorité vantée de Spallart, ${ }^{1}$ est un article de Michel Chevalier dans la Revue des Deux-Mondes ${ }^{2}$ dont il nous suffira de citer un passage essentiel: 'c'est exagérer, selon toute apparence, la production totale de la France en produits matériels que de la mettre à 10 milliards. Supposons que demain par un décret révolutionnaire, on installe le système communiste en France, ... . et que chaun des 35 millions de Français ait à prendre son lot égal sur les 10 milliards: ce sera par tête 78 centimes à dépenser par jour. Chaque ouvrier non marié sera mis à 78 centimes: je n'en sache pas beaucoup à Paris qui se contenteraient de ce traitement-là, même au nom de la République . . .'

Pareille méprise n'est pas heureusement très fréquente: 1' 'estimation' que nous venons de citer est probablement de beaucoup la moins solide que nous ayons rencontrée. Il ne nous est pas interdit d'ailleurs de la conserver dans notre collection et de comparer cette impression d'un contemporain avec des évaluations plus sérieusement justifiées-il n'est pas indifférent de savoir en quelle mesure 1'opinion concordait ou non, à I'époque, avec les statistiques valables.

Outre les erreurs assez grossières dont nous venons de donner quelques exemples, il importe de signaler aussi l'attribution de l'étiquette: Revenu ou Produit National, à des sommes globales qui ne la méritent guère ou qui même n'y prétendaient pas dans 1'esprit du calculateur original. Ainsi le chiffre de 11.3 milliards donné par Clark pour 1860 est une conversion en francs des 450 millions de livres sterling que Leone Levi attribue au Revenu français ${ }^{3}$ et qu'il a lui-même tiré, par conversion, des 11.121 millions de francs que Maurice Block attribue à la production industrielle totale; la confusion vient de ce que Block oppose sa propre estimation à celle de la statistique officielle qui ne concernait que la grande industrie. L'erreur de Levi sera répétée un peu plus tard par Edouard Vignes qui cherche en 1864 à obtenir une somme de revenus distribués égale à 11 milliards. Vignes est d'ailleurs récidiviste: dans son Traité des Impôts ${ }^{4}$ souvent cité, il donne pêle-mêle des chiffres globaux concernant

${ }^{x}$ Pour être juste, reconnaissons que Clark refuse toute signification aux évaluations antérieures à 1860. Mais c'est justement ce que nous contesterons: les chiffres de la fin du siècle ne nous semblent pas les meilleurs.

' Michel Chevalier, Question des Travailleurs, Revue des Mondes, 15 Mars, 1848 , p. 1067.

Leone Levi, Journals of the Royal Statistical Society, 1860, p. 42.

4 Vignes, Traité des impôts en France, Paris, 1880. 
toutes sortes de catégories distinctes (revenus imposables, produit net du sol, production industrielle, etc.) et sa liste recopiée par Foville a souvent passé toute entière chez les auteurs postérieurs sauf chez ceux qui ont tout rejeté par impatience ou mauvaise humeur; il vaut mieux faire un tri, et c'est presque toujours possible. Il est clair, par exemple, que les 6 milliards ${ }^{1}$ d'Hippolyte Passy, (projet de loi, 1849) ne correspondent qu'à une assiette possible d'un futur impôt et non pas à un produit ni à la masse du revenu distribué. Les chiffres partiels, pourvu qu'ils soient expliqués et justifiés ne sont pas du tout négligeables pour notre but; il convient seulement d'éviter les confusions.

3. Commençons par mettre à part les sources de documentation incomplète pour constituer ensuite une liste des estimations globales qui pourraient concourir à donner une idée de la croissance du produit national français. On trouvera dans la Note $\mathrm{A}^{2}$ l'inventaire des sources incomplètes, dont quelques-unes sont très importantes et ont été utilisées par la suite. Dans notre premier essai de collection, nous avons décidé d'être aussi peu sévères que possible, acceptant toute estimation, sous bénéfice d'inventaire, pourvu qu'elle se présente avec le minimum de garanties: nous avons dû évidemment tenir compte de la personnalité de l'auteur, des sources qu'il cite ou que 1'on peut présumer qu'il a connues, et surtout du fait que toute évaluation globale doit être présentée comme une somme d'éléments déterminés. Il ne s'agit cependant pas d'un palmarès: seule une étude ultérieure approfondie permettra de porter un jugement sur la réalisation concrète des intentions propres de chaque auteur. Pour le moment, nous nous contentons d'enregistrer et de comparer.

La comparaison soulève immédiatement un problème difficile: on peut définir un Revenu National de bien des manières. ${ }^{3}$ Plutôt que de tenter une uniformisation arbitraire, nous avons préféré effectuer la comparaison en deux étapes. Dans la première, nous étudions uniquement les séries ou chaînes d'évaluations faites par le même auteur pour diverses époques.

Ainsi que nous l'avons déjà dit, nous ne pensons pas que 1'étude de la croissance du chiffre global du Produit National soit d'un grand secours pour l'interprétation économique-aussi

${ }^{\imath}$ Cités par Vignes, puis Foville, puis Clark.

p. 58 .

See Note B, p. 61: 
notre projet comporte l'établissement d'une chaîne de comptabilités nationales tout au long de la période étudiée. Cette chaîne est en cours d'élaboration, les premiers résultats provisoires sont donnés dans la section III. Nous lui empruntons ici trois chiffres globaux: 1788, 1845, 1885 que nous comparerons aux données recueillies.

4. La question du taux de croissance du Produit National a été posée par un certain nombre d'auteurs: plus de la moitié des estimations dont nous pouvons disposer font partie de séries ou chaînes qui présentent pour nous un intérêt capital. Nous commençons donc par rassembler et comparer les estimations liées dans le temps, le Tableau 1 donne les chiffres et les sources: 35 estimations faites par 10 auteurs. Le diagramme 1 figure les évolutions correspondantes: l'échelle des valeurs (graduée en milliards de francs or) est logarithmique et choisie de façon que $l^{\prime}$ inclinaison à $45^{\circ}$ corresponde à peu près à un taux de croissance de $2 \%$ par an, doublement en 35 ans. Comme il fallait s'y attendre, les divergences de points de vue influent notablement sur les valeurs absolues des résultats.

Mais ce qui apparaît très nettement, par contre, c'est un accord remarquable pour ce qui concerne 1'allure générale de 1'évolution: ce qui se marque sur le diagramme logarithmique par le parallélisme des chaînes. On est amené à penser que diverses définitions du Revenu National peuvent évoluer d'une façon grossièrement proportionnelle: ce qui s'accorde avec le fait que les décompositions principales d'une comptabilité nationale ne font apparaître que de très faibles et très lentes variations des proportions.

On pourrait même effectuer pour chaque chaîne une translation de façon à obtenir un groupement optimum des 35 points du diagramme. Une opération graphique suffit, dont le résultat est donné par le diagramme 2 . Cette opération équivaut à une détermination analytique d'un taux typique de croissance autour duquel se distribuent les diverses estimations. On vérifierait sans peine que l'ajustement analytique revient à faire une moyenne pondérée des taux de croissance résultant des estimations individuelles de chaque auteur (Cf. Tableau 2).

On peut donc dire que la croissance du Revenu National a été, en gros, géométrique entre 1789 et 1914 . Le taux moyen est un peu inférieur à $2 \%$ par an. (Taux annuel: 1,8 ; ou $20 \%$ 
par décade.) Ce qui correspond à 'opinion reçuse $^{1} \mathrm{Si}$ l'on veut décrire l'évolution du Revenu National d'après les données que nous avons produites ci-dessus, on dira simplement que le Revenu National a doublé en 39 ans ou a été multiplié par 6 au bout de 100 ans. Peut-on raffiner et donne une description des fluctuations? Il ne nous semble pas. Si, comme nous l'avons fait sur le diagramme 2, on enferme tous les points représentatifs dans une bande rectiligne, 1 'épaisseur de cette bande correspond à une incertitude de $15 \%$ en plus ou en moins. Or, on ne peut espérer que les erreurs inévitables de chaque estimation aient toujours été notablement inférieures à ce chiffre: on peut même avancer que dans tous les cas l'erreur à craindre est de cet ordre. Il ne nous paraît donc pas possible de considérer comme significatives les ondulations qu'on aperçoit sur le diagramme, malgré la tentation qu'on aurait d'y voir des cycles longs.

5. Toutes les évaluations dont nous avons fait état jusqu'ici sont esprimées en francs courants; chaque fois que l'on doit interpréter une série de chiffres décrivant l'évolution en termes monétaires d'une certaine quantité globale, revenu ou produit, on a l'habitude de dissocier deux composantes, l'une purement monétaire, l'autre que 1'on dit 'réelle'. Il est certain qu'une hausse des prix ne doit pas être assimilée à une véritable croissance. Mais il importe de noter, pour le cas qui nous occupe, que jusqu'à 1914 les fluctuations de prix ont été relativement peu importantes. Sur le diagramme 3 ont été figurés, à l'échelle même des diagrammes 1 et 2 , quelques indicateurs de la valeur de la monnaie: indices des prix, indices du coût de la vie. Compte tenu des incertitudes dont il a été question au paragraphe précédent, on voit que la réduction des chiffres bruts au moyen d'un indice monétaire n'aurait ici qu'un effet minime ct probablement assez peu significatif. Si l'on tente cependant cette réduction, par exemple sur les données du diagramme 2 , on obtient une légère diminution du taux moyen de croissance.

Par contre, pour prolonger l'étude au delà de 1914, il devient absolument nécessaire de tenir compte de la valeur de la monnaie. Dans le diagranme 4 nous avons raccordé les données antérieures à 1914 avec celles calculées en termes 'réels' par Vincent et Froment, d'après les estimations de Colson et Dugé de Bernonville. Il semble alors, mais il est peut-être prématuré

${ }^{I}$ See Sauvy, Richesse et Population, 2ième edition, p. 93. 
de conclure, que le rythme général de croissance, commencé au moins à la fin du 18ème siècle, se soit assez profondément modifié après 1914.

Les données numériques utilisées pour la confection des diagrammes 3 et 4 figurent dans le Tableau 3. On a figuré, sur le diagramme 4, le mouvement de la population. On constate que ce mouvement a été très lent au cours de la période étudiée. Si l'on voulait calculer, comme de coutume, le revenu 'par tête', les résultats seraient peu modifiés, et on n'éclairerait guère les relations entre la croissance économique et la croissance démographique. On s'étonnera évidemment de voir que ces relations sont très peu apparentes. A l'échelle où nous opérons, la régularité de la croissance du revenu semble complètement indépendante d'une foule de circonstances, politiques ou autres, qui ont beaucoup varié depuis 1780 !

6. Après avoir rassemblé les évaluations liées, nous devons leur comparer les évaluations isolées: c'est la deuxième étape annoncée ci-dessus. Le Tableau 4 rassemble 15 évaluations et le diagramme 5 les situe par rapport aux chaînes déjà présentées. Comme il a été convenu, nous faisons figurer aussi bien les évaluations solides et détaillées que d'autres beaucoup plus fragiles: on constatera cependant que la dispersion n'augmente guère. Il semble, par conséquent, que s'il ne s'agissait que de mettre en lumière le fait de la croissance et d'estimer un taux moyen, la précision fournie par nos sources serait tout à fait suffisante.

S'il s'agit, comme nous le pensons, d'interpréter le phénomène, alors la difficulté principale proviendra de la finesse nécessaire aux estimations statistiques: les phénomènes essentiels jouent à l'intérieur d'une marge qui, pour le moment, est comprise dans la marge d'incertitude générale. 
TABLEAU 1

(Estimations liées du Revenu National Annuel en millions)

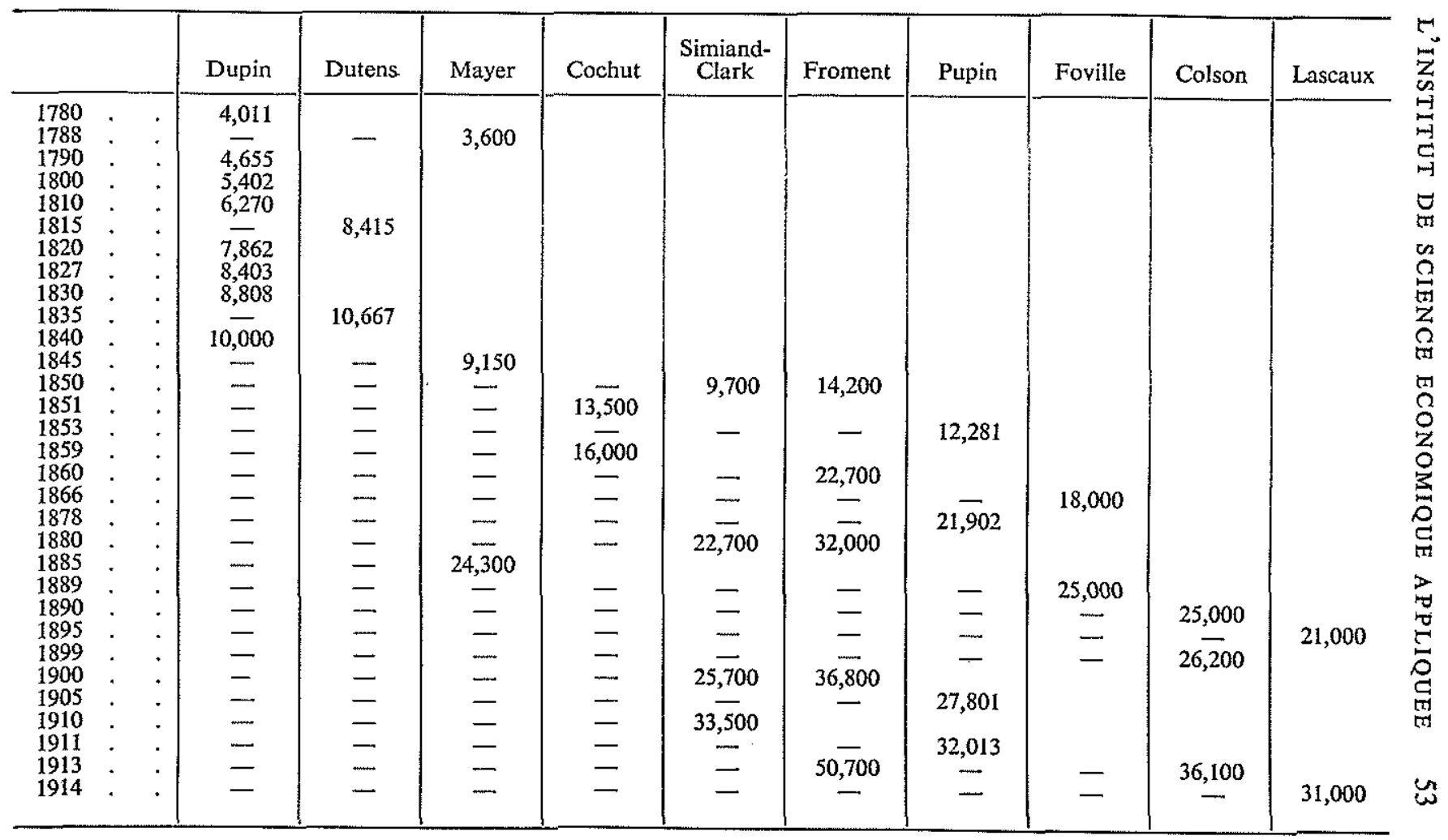




\section{NO'TES DU TABLEAU 1}

DUPIN

Voir Ch. Dupin, Forces productives et sociales de la France, 2 vol., Paris, 1827.

Ch. Dupin, Bien-étre et concorde des classes du peuple français, 1848 (Petits Traités publiés par l'Acad. Sc. Morales et Politiques, $\left.n^{\circ} 12\right), 141 \mathrm{pp}$.

J. H. Schnitzler, Statistique générale, méthodique et complète de la France, Paris, 1846, 4 vol.

DUTENS

Joseph Dutens, Essai comparatif sur la formation et la distribution du Revenue de la France en 1815 et en 1835, Paris, 1842.

MAYER

Cf. ci-après Section III.

\section{COCHUT}

André Cochut, Philosophie de l'économie politique, Revue des Deux-Mondes, 1er avril 1859.

\section{SIMIAND-CLARK}

Les indices donnés par Simiand (Le Salaire, Paris; 1932, Tome 3, p. 107) ont été transformés en valeurs monétaires par Clark (The Conditions of Economic Progress, 2e ed., pp. 71 et 74).

\section{FROMENT}

René Froment, Trois journẻes pour l'étude scientifique du vieillissement de la population (avril 1948), Alliance Nationale contre la dépopulation, Paris, 1948, Fascicule IV, pp. 41 sqq.: La part des vieillards dans le Revenu national, pp. $42-43$.

Cf. Fourastié, Machinisme et Bien-être, Paris, 1951, p. 96, note 2.

\section{Pupin}

R. Pupin, de la Richesse de la France devant la Guerre, Paris, 1916.

\section{Foville}

Dictionnaire des Finances publié sous la direction de Léon Say, 1883-1894; Article Richesse, pp. 103 sqq., par A. de Foville.

Cf. Levasseur, Questions ouvrières, Paris, 1907.

COLSON

C. Colson, Cours d'Économie Politique, Livre III, Paris, Gauthier-Villars, 1927, pp. 387-404.

\section{LASCAUX}

Robert Lascaux, La production et la population, Paris, Payot, 1921, pp. 251254. 


\section{TABLEAU 2}

\section{Taux de croissance du Revenu National selon les évaluations du Tableau 1}

$\left.1^{\circ}\right)$ Taux individuels

On remarquera que les extrêmes correspondent à des chaînes courtes.

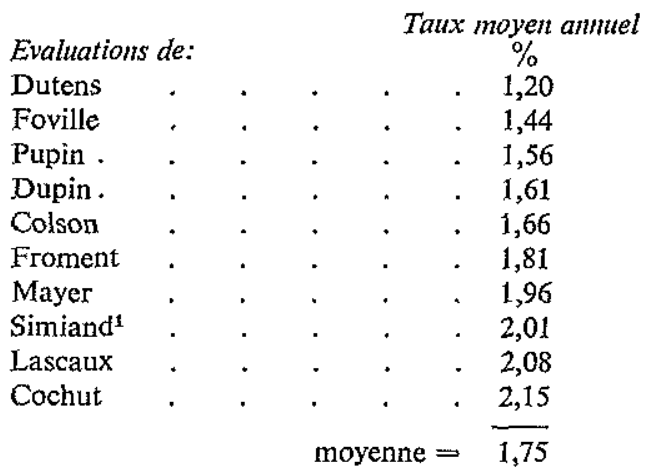

$2^{\circ}$ ) Estimation d'un Taux Moyen Général

On trouve: Taux $=1,81 \%$ par an.

\section{TABLEAU 3}

1. Dans le diagramme 3 l'indice des prix de gros est celui des 45 articles publiés par la Statistique Générale de la France. Ceux du coût de la vie sont

A): indice de March (dans: Salaires et cout de l'existence à diverses époques jusqu'en 1910; Paris, 1911, p. 105) reproduit par Sauvy, op. cit., 2e ed., p. 71, et cité par Clark, op. cit., 2e ed., p. 76.

B): indice calculé par Portzamparc et Froment (dans: Institut de Conjoncture, $L$ 'intérêt réel du capital, Etude spéciale $\mathrm{N}^{\circ} 1$ );

C): indice calculé par Chabert, Essai sur les mouvements des revents et l'activité économique en France de 1798 à 1820, Paris, 1949, p. 264 (si l'on compare cet indice à ceux de Simiand, op. cit., Tome 3, p. 88, on verra qu'il s'agit bien plus d'un indice des prix que du coût de la vie proprement dit).

2. Les chiffres du Revenu National postérieurs à 1920 sont empruntés à l'Étude Spéciale $\mathrm{N}^{\circ} 3$ de 1'Institut de Conjoncture: Le progrès technique en France depuis Cemt Ans, Paris, 1944, Tableaux 20 et 32, pp. 109 et 122. Les donnés de base sont les évaluations de Colson et de Dugé de Bernonville, - réduites au moyen de l'indice (B) cité plus haut.

${ }^{1}$ La transformation de Clark n'est pas nécessaire; on peut ici traiter directement les indices de Simiand. 
Tableau 3 (suite)

Voici les résultats (indice du Revenu National réel):

$$
\begin{aligned}
& 1901=100 \\
& 1913=126,1 \\
& 1920=114,0 \\
& 1921=131,6 \\
& 1922=142,8 \\
& 1923=140,4 \\
& 1924=148,5
\end{aligned}
$$

\begin{tabular}{|c|c|c|c|c|}
\hline 1788. & Tolosan. & . & . & - 3 milliards (produits) \\
\hline 1789. & Neymarck & . & , & entre 3 et 5 \\
\hline 1791. & Renard . & . & . & 5,1 (consommation) \\
\hline $1804-10$. & Chaptal & . & . & . 6,1 (produits) \\
\hline 1812. & Montalivet & . & . & . 7,0 (idem) \\
\hline 1830. & Fourastié & . & - & . 8 \\
\hline 1842. & Schnitzler & . & . & 7,7 (produits) \\
\hline 1848. & Chevalier & . & . & 10 \\
\hline 1868. & Ayen . & . & $\cdot$ & - 18 à 20 (revenus privés) \\
\hline $1870-74$ & Wolowski & . & & . 20 à 22 \\
\hline 1877. & Leroy-Beaulie & & & . 25 \\
\hline 1883. & Cochut & . & & 37,1 \\
\hline 1889. & Neymarck & . & & . $\quad$ entre 30 et 35 \\
\hline 1890. & Coste & & • & 22,5 \\
\hline 1905. & Lavergne et $\mathrm{H}$ & Henry & & 27,8 \\
\hline
\end{tabular}

$$
\begin{aligned}
& 1925=152,1 \\
& 1926=146,0 \\
& 1927=145,0 \\
& 1928=155,1 \\
& 1929=155,8 \\
& 1935=127,1 \\
& 1936=139,6 \\
& 1937=137,8 \\
& 1938=134,8
\end{aligned}
$$$$
1930=147,3
$$$$
1931=141,0
$$$$
1932=137,7
$$

\section{TABLEAU 4}




\section{NOTES DU TABLEAU 4}

TOLOSAN

Mémoire sur le commerce de la France et des Colonies, Paris, in- $8^{\circ}, 1789$ (publié sans nom d'auteur) (Bibl. Nationale: $V, 17.751$ ).

\section{NEYMARCK}

Un centenaire économique, Journal de la Société de Statistique de Paris, 1889.

\section{RENARD}

Découverte de la valeur certaine du produit réel et absolu du sol de la France ...par le sieur Renard (Bibliothèque du Ministère des Finances, Recueil, 12-3620).

\section{CHAPTAL}

De l'industrie française, Paris, 2 vol., in $8^{\circ}, 1819$.

\section{Montaliver}

Ancienne et nouvelle France, Paris, 1813.

\section{FOURASTIÉ}

Machinisme et Bien-Etre, Paris, 1951, pp. 90 et 96, note 2.

\section{SCHNITZLER}

Statistique générale, méthodique et complète de la France, Paris, 1846, 4 vol.

\section{Chevalier}

Questions des travailleurs, Revue des Deux-Mondes, 15 mars 1848.

\section{AYEN}

Journal des Économistes, 1875, p. 236.

\section{WoLOWSKI}

Cité par A. de Foville, La Richesse de la France (Extrait du dictionnaire des Finances de Say); cf. aussi Rouvier, discours du 3 janvier 1874 .

\section{LEROY-BEAULIEU}

Cité par Levasseur, Questions ouvrières, Paris, 1907.

\section{CochuT}

De l'enchérissement des marchandises et des services, Revue des Denx-Mondes, ler décembre 1883 , pp. 512-551.

\section{Coste}

Étude sur le salaire des travailleurs et le Revenu de la France, Journal de la Société de Statistique de Paris, 1890.

\section{LAVERGNE}

Lavergne et Henry, La Richesse de la France, Paris, 1908. 
NOTE A

Evaluations qui n'ont pas été prises en considération dans l'étude précédente

\section{LAVOISIER}

Lavoisier avait entrepris un ouvrage 'de la Richesse territoriale du Royaume de France' dont la rédaction ne fut, semble-t-il, jamais terminée, mais dont nous possédons quelques éléments par: Résultats extraits d'un ouvrage intitulé: De la Richesse territoriale du Royaume de France, ouvrage dont la rédaction n'est point encore achevée; imprimé sur l'ordre de l'Assemblée Nationale, Paris, 1791. Cet opuscule a été réimprimé dans la Collection des Principaux Économistes, par E. Daire et S. de Molinari, Paris, Guillaumin, Tome 14, pp. 575 sqq., ainsi que dans les Oeuvres de Lavoisier, 6 vol., in $4^{\circ}$, Imprimerie impériale, Paris, 1864, Tome 6, pp. 403463. Son importance est exceptionnelle, tant par les renseignements statistiques qu'il apporte, que par les conceptions théoriques (d'inspiration physiocratique) qu'il illustre, ef les méthodes statistiques. Roederer, dans l'avertissement, signale que les commissions parlementaires de l'époque ont trouvé que les chiffres de Lavoisier étaient sous-estimés. Lavoisier s'efforce de chiffrer le Revenu Territorial, c'est-à-dire à peu près le produit brut de l'agriculture, qu'il évalue à 2.750 millions. Le produit net, c'est-à-dire le précédent diminué de la consommation des cultivateurs et auxiliaires, est de 1.200 millions. Les décompositions et les divers éléments statistiques allégués sont, pour nous, plus importants que ces sommes globales.

Cf. aussi, le travail de Lagrange sur les Besoins de la Nation, (Collection des Principaux Économistes).

\section{P. DE Delay (d'Agier)}

Député du Dauphiné à la Constituante qui en 1790, à I'occasion des réformes fiscales, a présenté dans une série d'Opinions, prononcées à l'Assemblée, quelques estimations assez détaillées des revenus fonciers et mobiliers.

Selon Delay, les revenus fonciers sont à peu près équivalents aux mobiliers, et chacune des deux sommes dépasse peu le milliard.

Il est cité par:

- Vignes, Traité des impôts, op. cit., 4e édition, tome 2, p. 141, d'aprés Deparieu.

- Foville, La Richesse de la France et la France Economique, op. cit., d'après Vignes.

- Clark (sous le nom: 'D'Agier'), dans 'The Conditions', op. cit., d'après Foville.

${ }^{`}$ On écrit aussi Dedelay, Dedelley, Delai. 
Ses estimations peuvent être rapprochées de celles de Lavoisier pour les produits agricoles. Elles comportent des chiffres pour les loyers urbains; les salaires publics, la dette publique. Ce sont ces chiffres partiels qui sont les plus utiles. La somme totale (que donne Clark) ne représente nullement un produit national: 1'intention de l'auteur reste essentiellement fiscale.

\section{J. B. E. Poussielgue}

Cet inspecteur des finances a écrit un ouvrage: Des finances de la France en 1817 qui comporte diverses estimations globales (Revenus fonciers, revenus mobiliers, etc.), en particulier celle du total des revenus imposables (3,7 milliards). Ce sont ses chiffres, d'optique proprement fiscale, qui seront reproduits par Foville (dans 'La Richesse de la France', extrait du Dictionnaire des Finances, ParisNancy, 1893) et cités par Clark.

\section{Passy et GoudchauX}

Dans son Traité des Impôts, Edouard Vignes cite:

- Hippolyte Passy: en 1849, exposé des motifs du projet de loi relatif à l'impôt sur le revenu;

Revenu total imposable (foncier et mobilier) au minimum de 6 milliards.

- Goudchaux (ministre des Finances), projet d'impôt sur les revenus mobiliers (1848), Revenus mobiliers s'élèvent à 3 ou 4 milliards (chiffre adopté par la commission).

Cis deux chiffres ont été transmis par Foville à Clark.

\section{CoCHUT}

Dáns un article de la Revue des Deux-Mondes, janvier 1849, A. Cochut estime les revenus mobiliers à 3.137 millions. Même en y ajoutant les revenus fonciers (comme fait Clark d'après Foville) il y manquierait encore les salaires.

\section{Ballue et Peyral}

Cités par Foville (Dictionnaire de Say, et: France É conomique):

- Ballue (Rapport à la chambre, 26/xI/1886): 8 milliards;

- Peyral ou Peytral (projet de loi du 30/x/1888): 16 milliards; il s'agit encore ici de revenus imposables. Foville se contente de noter que ces chiffres sont inférieurs au revenu national.

\section{VIGNES}

Dans son Traité des Impôts, après avoir cité l'ouvrage de Maurice Block (cf. infra $\mathrm{n}^{\circ} 10$ ), Vignes cherche à faire la somme des revenus 
distribués pour obtenir les onze milliards de Block. Il y a vraisemblablement sous-estimation des salaires (calculés d'après le recensement de 1851 et le salaire moyen de 1858), ainsi que des traitements des employés et des professions libérales. D'ailleurs le chiffre de Block ne concerne que la production industrielle.

\section{MONDENARD}

Le marquis de Mondenard, Considérations sur l'organisation sociale, 3 vol., 1802. Cite les estimations de J. Bosc pour 1789, assez peu différentes de celles de Tolosan.

\section{GUILHAUd DE LAVERGNE}

Économie Rurale de la France depuis 1789, Paris, 1877. L'auteur reprend les estimations de Lavoisier, Chaptal, Moreau de Jonnès, et cherche à les améliorer.

\section{Maurice BLocK}

Statistique de la France (Paris, Guillaumin, 1ère éd. 1860, 2e éd. 1875). Cet ouvrage contient (pour 1860 et pour 1872) les éléments d'une comptabilité nationale très détaillée. Il est à rapprocher des ouvrages similaires de Schnitzler et de Moreau de Jonnès. Nous avons déjà expliqué pourquoi l'usage qu'en font Leone Levi et Edouard Vignes nous paraît inacceptable.

11. N'ont pas été examinés:

- de Parieu, cité sans référence par Vignes et Foville;

- Gaudin, 1817, cité par Foville;

- Teisserenc de Bort, Assemblée Nationale, 22/xIr/1871;

- Troplong, Petits traités (Paris, Guillaumin);

- Dictionnaire du commerce publié sous la direction de Guillaumin. 


\section{NOTE B}

Il faut noter ici que l'optique des évaluations et la nature de la documentation ont varié au cours des autres périodes. Il semble qu'il $y$ ait des relations certaines entre l'état de l'économie à une époque et les préoccupations et la documentation qui caractérisent cette époque.

Au XVIIIe siècle, 1'agriculture constitue, de beaucoup, le secteur fondamental de l'économie. C'est de lui essentiellement que paraît dépendre tout le progrès ultérieur. Ce fait et cette croyance apparaissent dans les théories des Physiocrates. Elles influencent d'une façon décisive les évaluations.

Au milieu du XIXe siècle, la France est en train de connaître la révolution industrielle. Annoncée depuis quelques décades, elle est encore loin d'avoir pris son plein essor. On a conscience de son importance pour la production des richesses. J. B. Say, Saint-Simon, les économistes libéraux en entretiennent l'opinion. A côtẻ d'une enquête agricole, on recensera donc soigneusement les établissements industriels de quelque importance. La tâche est jugée nécessaire; elle n'est pas encore très difficile.

Après 1880, la documentation concernant le produit national apparaît relativement plus pauvre. Sans doute, le dénombrement d'une économie plus tiche, plus diversiffée, serait-il plus difficile. Les auteurs supputent surtout les revenus privés.

Il semble donc que l'optique et la documentation aient évolué, grosso modo, des produits aux revenus: du produit agricole, au début de beaucoup le principal, et qui fait la base de la prospérité, aux produits agricoles et industriels ensuite, dont on sait l'importance pour les progrès ultérieurs et dont le dénombrement reste assez aisé, aux revenus enfin, en liaison avec des problèmes de finances publiques et de répartition. C'est une autre source de différences entre les estimations. Ce n'est pas seulement la chose observée qui change, mais ceux même qui l'observent, la façon de voir des observateurs. Les estimations et la doctumentation ne sont pas neutres. 
62

INCOME AND WEALTH

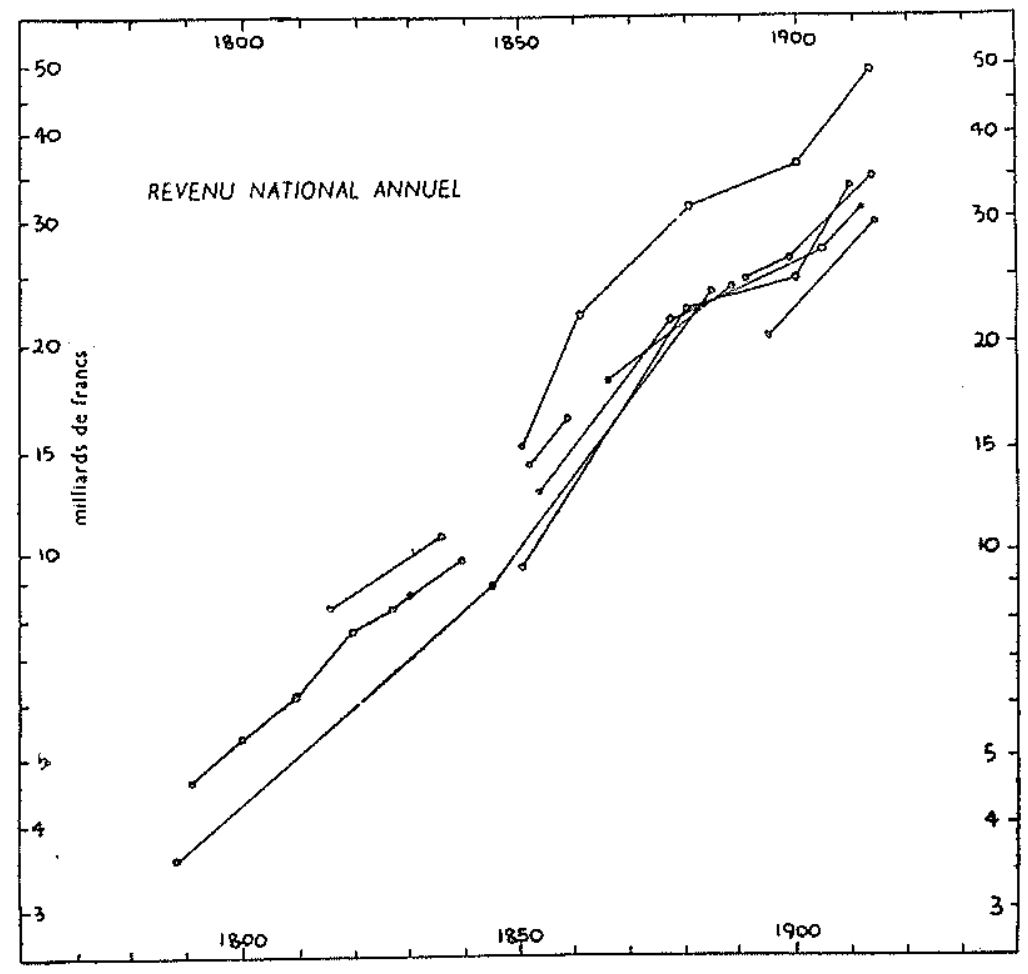

Diagramme 1 


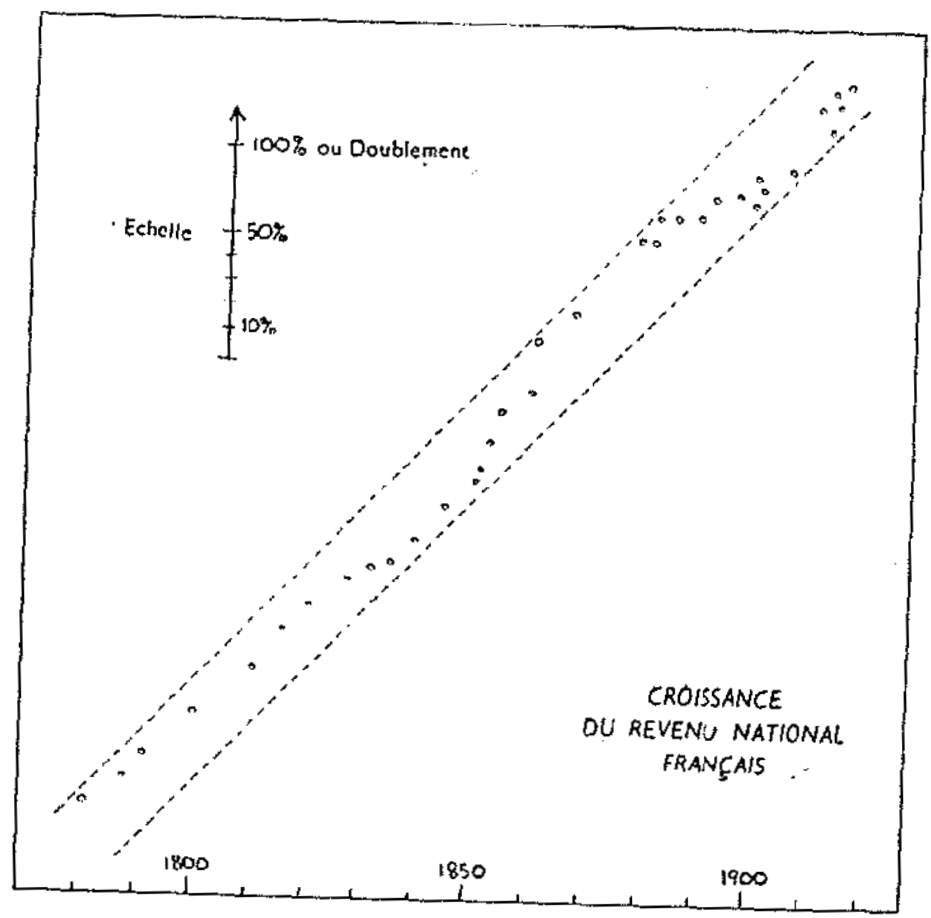

Diagramme 2 


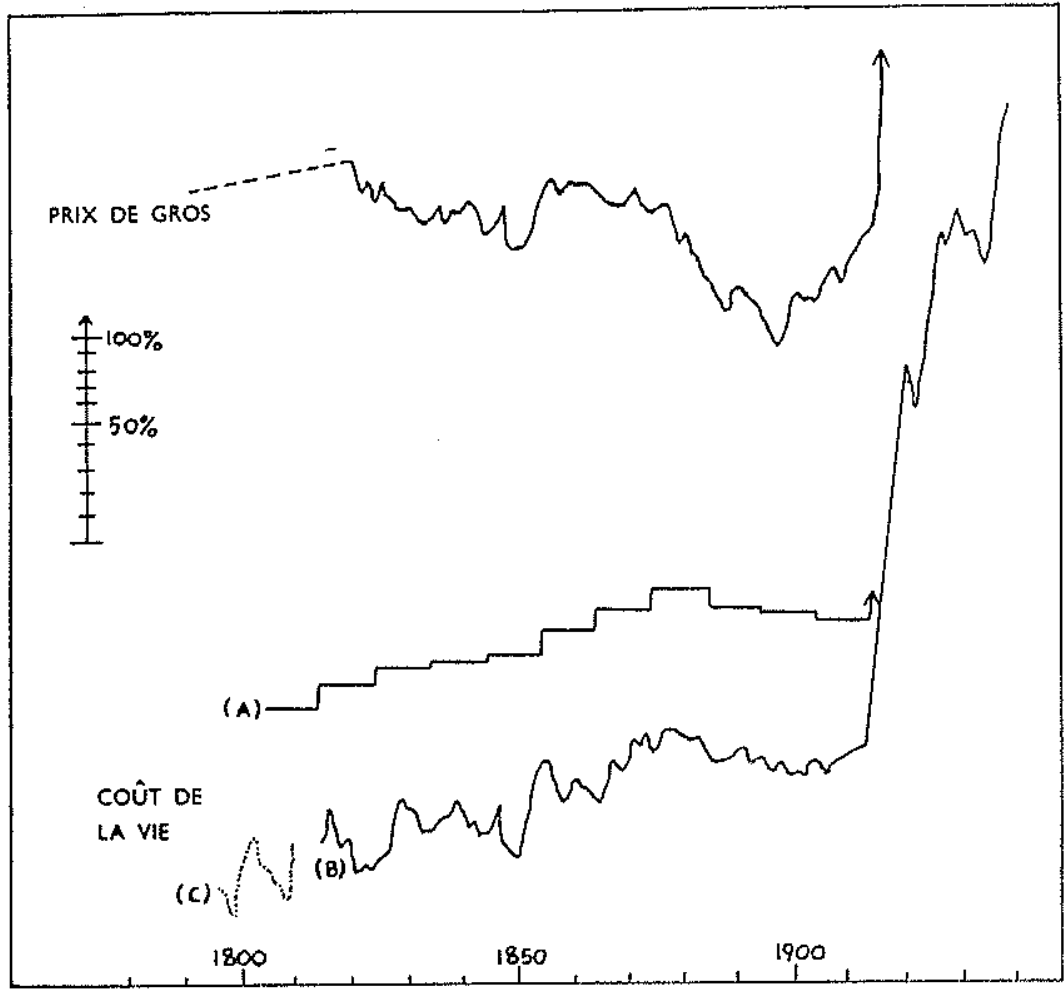

Diagramme 3 
L'INSTITUT DE SCIENCE ECONOMIQUE APPLIQUEE 65

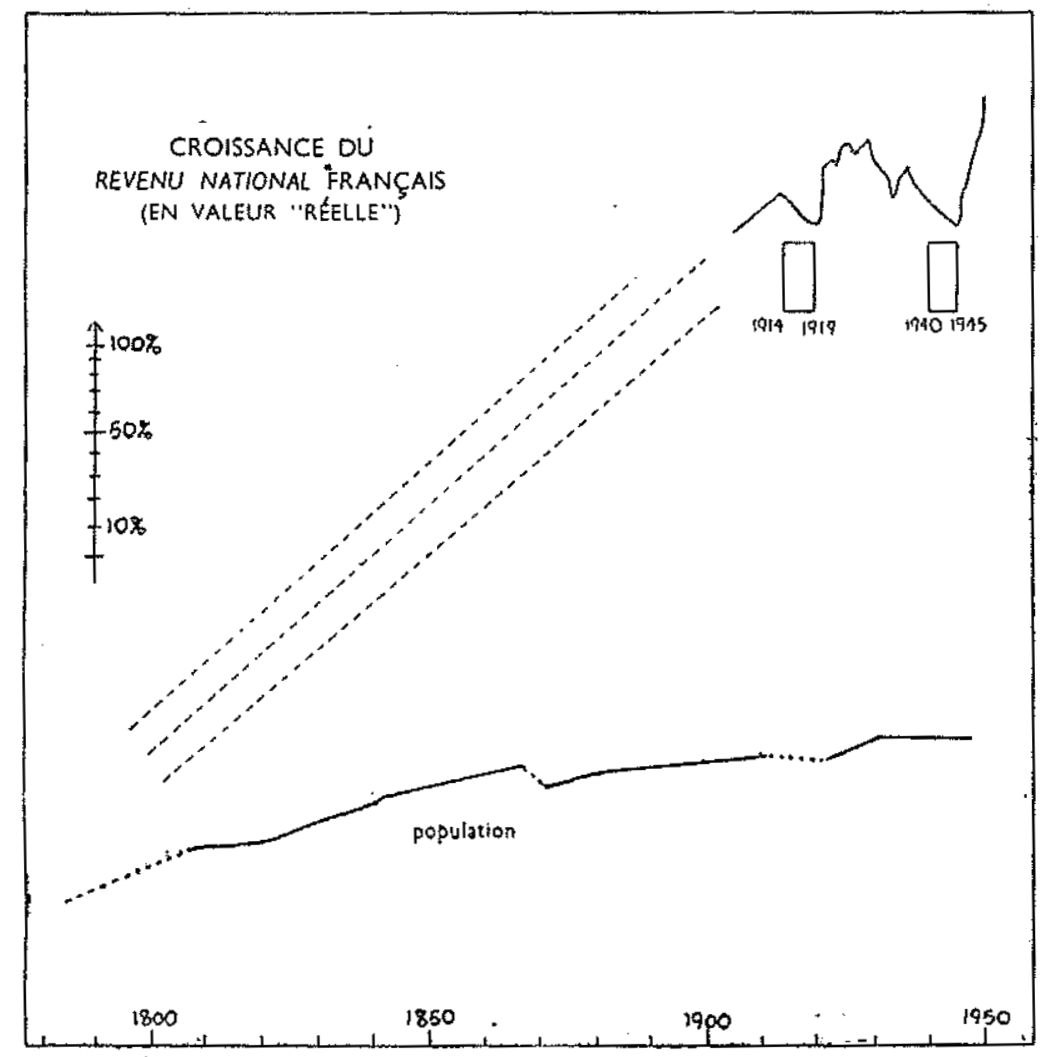

Diagramme 4 


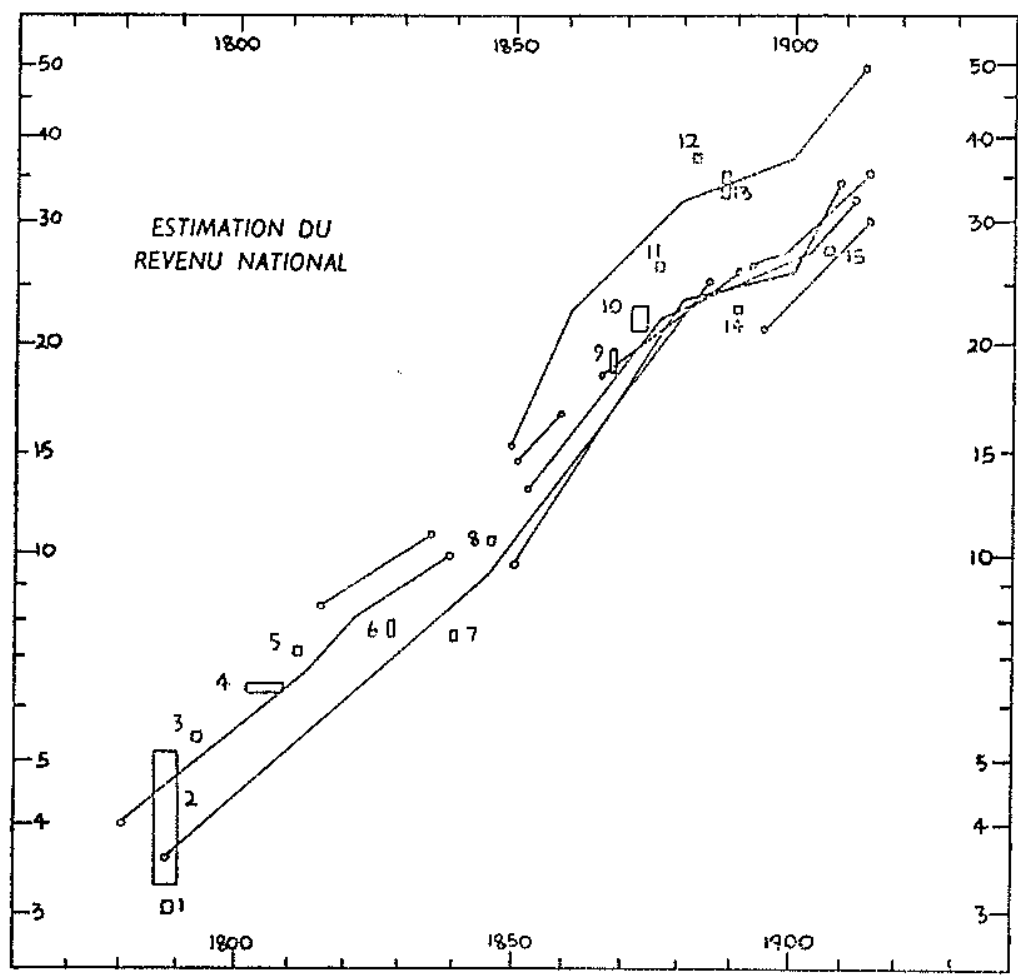

1. Tolosan 4. Chaptal 7. Schnitzier 10. Wolowski 13. Neymarck

2. Neymarck 5. Montalivet 8. Chevalier 11. Leroy-Beaulieu 14. Coste
3. Renard
6. Fourastié
9. Ayen
12. Cochut
15. Lavergne

Diagramme 5 
III. LA STRUCTURE DE L'ÉCONOMIE FRANÇAISE

à trois époques éloignées: 1788, 1845, 1885

par J. Mayer

\section{Introduction}

En partant des documents statistiques de quelques époques caractéristiques de notre histoire économique, nous voulons rassembler des données aussi précises que possible sur la structure économique de la France, et dresser une comptabilité nationale sommaire pour ces trois époques.

Nous avons choisi des époques qui satisfont à deux conditions: marquer des changements de structure économique et avoir été étudiées dans d'assez nombreux documents statistiques.

La période qui précéda immédiatement la Révolution de 1789 satisfait ces deux conditions: c'est en effet l'époque des travaux de Lavoisier, Tolosan, Delay.

Ces deux conditions se sont trouvées également réunies dans la période 1845-1848. Les évaluations connues du revenu national français matquent en effet un point de discontinuité à cette époque ainsi que des séries aussi caractéristiques que celles de la consommation de houille ou de la longueur dú réseau ferré. D'autre part, la Statistique de la France entreprenait alors ses premières enquêtes, véritables recensements de la production.

La date 1885-1886 s'impose moins nettement. Du point de vue cyclique, c'est une période de reprise économique. Enfin, c'est une période de multiplication des études statistiques.

Nous ne pouvons aborder avec quelques chances de succès l'étude des documents sans nous livrer à une courte réflexion sur les vues de leurs auteurs. Structure économique, théorie des économistes et recherches des statisticiens sont des choses trop étroitement liées pour que nous puissions utiliser ces dernières sans nous préoccuper des précédentes.

Les théories prédominantes en France au cours du XVIII ${ }^{\circ}$ siècle étaient celles des physiocrates. A une époque où la classe politiquement dominante, la noblesse, et l'Etat tiraient la plus grande part de leurs ressources des revenues fonciers et des 
impôts sur les produits du sol, les économistes durent expliquer comment, seuls de tous les travailleurs, les paysans produisaient de quoi subvenir à leur propre consommation et à celle d'autres personnes. Cette structure politique était, en gros, celle de la France pré-révolutionnaire et c'est pourquoi nous ne devons pas nous étonner de voir Lavoisier imbu de l'esprit des physiocrates. Mais comme il prétend calculer, non seulement le produit net du royaume (exchuant $\mathrm{du}$ produit brut la consommation des travailleurs) mais le produit total, il se trouve placé devant une contradiction. D'ailleurs, faute de possibilité d'enquête, Lavoisier (I) se place sous l'optique de la consommation. C'est de l'estimation du budget familial des diverses couches de la population qu'il tire son estimation du revenu national et il ne peut la contrôler par des estimations des productions et des consommations globales des divers produits agricoles ou autres. A la même époque, de Tolosan (II) ajoute à la valeur de la production agricole la valeur nette de la production non agricole. De tels travaux, plus conformes à l'économie de l'époque, préparent la diffusion en France de l'économie classique.

En 1845 nous nous trouvons placés devant une optique toute différente. C'est essentiellement sous l'angle de la production que se placent les statisticiens. L'ampleur de 1'appareil administratif napoléonien, la relative simplicité de la structure industrielle et la nécessité dans laquelle se trouvait la bourgeoisie française d'accroître sa production pour lutter contre la concurrence anglaise, donnèrent naissance à de remarquables travaux statistiques. La notion de valeur ajoutée par chaque industrie aux matières premières, le partage de cette valeur entre salaire et profit sont des notions claires. Par contre, on ne se préoccupe que des productions matérielles, la plupart des services (tels que les professions libérales) ne sont pas inclus dans le produit national.

En 1885, le tableau est encore différent, et c'est l'optique $d u$ revenu qui prévaut. La plupart des auteurs reculent devant les difficultés d'une évaluation directe de la production et étudient les revenus distribués. En conséquence, on met tous les revenus sur le même plan et nous voyons apparaître les revenus des professions libérales et ceux des domestiques. C'est pourquoi nous voyons disparaitre l'optique de la production, car l'idée moderne que produire, c'est produire de la satisfaction, ne semble pas avoir eu encore cours chez les statisticiens. 
Pour essayer d'avoir des données comparables nous nous sommes attachés à ajouter, dans les deux premières évaluations, les revenus des professions libérales et ceux des employés de l'État. Quant aux revenus du commerce, il n'est pas toujours facile de savoir s'ils sont inclus ou non dans les évaluations car nous ne savons pas toujours si les prix utilisés sont des prix à la production, des prix de gros ou des prix de détail. Nous avons essayé de les séparer.

Avant de passser à l'étude des trois époques choisies, nous devons remarquer que tout ce que nous allons dire concerne essentiellement les biens ou services apparaissant sur le marché. Or, la nature de ces biens a varié d'une époque à l'autre; les scrupules des auteurs contemporains, à propos des services rendus par la ménagère à son foyer auraient eu bien plus de raisons de s'exercer il y a 150 ans. Nous ne nous intéressons qu'à la structure des transactions où intervient l'argent.

Enfin, précisons qu'il ne s'agit, dans les pages qui vont suivre, que d'une étude sommaire dont une recherche plus approfondie pourra peut-être modifier les résultats. ${ }^{1}$ Notre conviction est que, tout au moins pour les deux dernières époques, les documents existant à Paris permettront une très bonne détermination de la structure économique de notre pays.

\section{L'Economie française en 1788}

Nos principales sources d'information ont été les ouvrages de Lavoisier (I) et de Tolosan (II); Braesch (III) nous a donné des renseignements sur les budgets, enfin nous avons trouvé chez divers auteurs des données plus qualitatives que quantitatives qui nous ont permis de préciser certains points.

Les estimations de Lavoisier et de Tolosan sont en général concordantes; là oû elles diffèrent nous nous sommes servis de préférence de celles de Tolosan qui semble avoir eu à sa disposition 1'appareil administratif du royaume.

a) Agriculture. La principale culture était celle des céréales; il s'en récoltait environ 140 millions de quintaux (le quintal pèse environ $49 \mathrm{Kg}$.) non compris les semences, mais compris les grains qui servent de matière première à l'industrie et à la nourriture des chevaux de troupe et de transports.

${ }^{1}$ Les recherches poursuivies à l'ISEA depuis que ces lignes ont été ècrites ont justifié cette rèserve. On en trouvera un compte rendu dans $L a$ Croissance du Revemu National Francais depuis 1870, Cahiers de l'ISEA, Série D, No. 7, 1952. 
La question du prix est délicate car les différents auteurs ne sont pas d'accord sur le prix moyen des grains. Nous adopterons un sou six deniers la livre comme prix moyen sur les marchés de gros et nous ajouterons une plus value de 150 millions pour tenir compte de la fabrication du pain. Ceci nous donne une somme de 1.200 millions de livres. ${ }^{1}$

On trouvera tableau I la valeur des principales productions agricoles dont l'ensemble se monte à 2.300 millions de livres. Cette valeur est nette des semences mais contient les achats à l'extérieur, essentiellement à l'artisanat des campagnes. Nous la considèrerons donc comme une valeur de la production du secteur agriculture-artisanat des campagne et en déduirons 200 millions comme valeur des achats aux autres secteurs.

TABLEAU I

Production agricole, 1788

(en millions de livres)

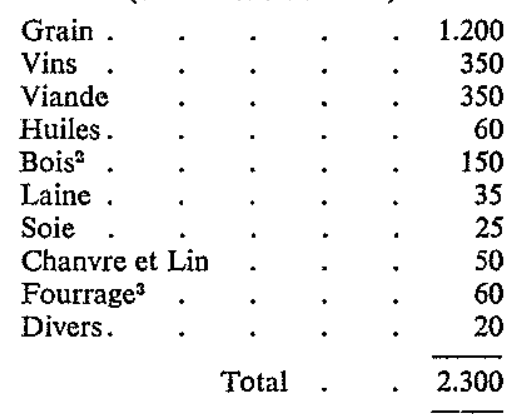

b) Industrie. Le tableau II donne les valeurs ajoutées par les différentes industries, extrait de Tolosan. Nous avons adopté le classement, que nous retrouverons à l'époque suivante, selon la nature de la matière première principale.

c) Services. Les données sont ici moins précises. L'ensemble des loyers urbains semble se monter à 250 millions (Lavoisier). Nous évaluerons à 100 millions le revenu des professions libérales, à 200 millions les salaires payés par l'État (sur un ensemble de dépenses budgétaires, dette non comprise, de l'ordre de 300 millions) et à 400 millions le revenu net du commerce et des transports. Le revenu national brut s'établit donc comme suit:

${ }^{1} \mathrm{La}$ transformation de la livre tournois en franc s'est faite à un taux très proche de la parité.

a Évaluation empruntée à Mirabeau (d'après Tolosan).

${ }^{3}$ Non compris le fourrage utilisé pour les animaux de labour et de boucherie. 

Agriculture et artisanat rural
2.100
Industrie et artisanat urbain
Commerce et transports
Loyers et professions libérales
Services de 1'État
550 Revenu national
$400\}$ brut: 3.600 millions
350 de livres
200

\section{TABLEAU II}

Valeur's ajoutées par les différentes industries

Produits minéraux

(en millions de livres)

Sel gemme et marin $(40.10 \mathrm{c} \mathrm{kg}$.) . . . . . 3

Faïence, porcelaine, verrerie. . . . . . 7

Métallurgie (production de fonte: $69.000 \mathrm{t}$ ) . . . 35

Quincaillerie, mercerie (chiffre très hypothétique) . . 75

Orfèverie, bijouterie . . . . . . . . 3

$$
\text { Total . . } \overline{123}
$$

Produits végétaux

Toile de chanvre, lin, coton . . . . . . . $\quad . \quad 160$

Amidon . . . . . . . . . 2

Savon . . . . . . . . . . . 5

Sucreries . . . . . . . . . .

Tabac . . . . . . . . . .

Papeterie, imprimerie . . . . . . . 27

$$
\text { Total . . . } \overrightarrow{200}
$$

\section{Produits animaux}

Lainages . . . . . . . . . 92

Soieries (étoffes) . $\quad . \quad$. $\quad . \quad . \quad . \quad . \quad . \quad . \quad . \quad 41$

Modes et tapisseries . $\quad . \quad$. $\quad . \quad$. $\quad$. $\quad$. 6

Tannerie . . . . . . . . . 6

Pêcheries . . . . . . . . . . . $\quad . \quad 10$

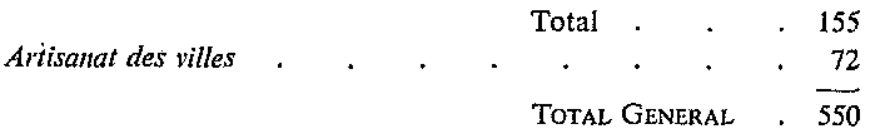

d) Revenu national net. Nous n'avons pas trouvé d'évaluation de l'amortissement. Nous considérerons ce revenu comme un revenu net, et par suite, 1'investissement dont nous allons parler sera un investissement net.

e) Utilisation du revenu national. Les chiffres que nous avons donnés ne se rapportent pas d'une façon précise à l'année 1788 . Ce sont des chiffres 'd'année moyenne' pour la période précédant immédiatement la Révolution de 1789. Au cours de cette 
période, la balance commerciale avec l'étranger, toujours très faible, oscillait autour de la moyenne zéro. Nous n'en tiendrons donc pas compte. Il nous reste à chercher comment ce revenu se partage entre consommation et investissement. A Paris, sur un revenu total de 300 millions, 40 étaient épargnés, ${ }^{1}$ soit $13 \%$.

En supposant que l'épargne était le seul fait des populations urbaines (y compris ceux qui vivaient du revenu de leurs terres) et en fixant sa valeur à $10 \%$ des revenus de ces populations, nous obtenons une valeur de 200 millions, soit environ $5 \%$ du revenu national. Nous partagerons cette quantité en deux, 100 millions étant consacrés à l'achat de biens à l'agriculture (dans laquelle s'est trouvée incluse la construction) et 100 à l'industrie. Le reste est consacré à la consommation de biens et services par les particuliers (3.000) et le gouvernement (400).

f) Partage entre les divers facteurs de production. Si nous retirons du revenu national les impôts indirects, il reste 3.300 millions à partager entre les facteurs de production.

Pour l'agriculture et l'artisanat rural, les données de Tolosan et Lavoisier permettent de fixer à 1.200 millions environ la part des travailleurs ruraux: ouvriers agricoles, fermiers, et métayers, petits propriétaires, artisans (c'est cette part que nous avons appelée 'salaire' dans le tableau). Le reste, soit 800 est le revenu des propriétaires.

Pour l'industrie et l'artisanat urbains, si l'on peut fixer à 100 millions la valeur des impôts indirects perçus sur leurs produits, la part des salaires est difficile à déterminer. Nous l'avons fixée à 300 millions en y incluant la rémunération du travail des petits patrons.

Pour les services, qui comprennet la location des habitations, nous avons fait la plus grande part au profit. Enfin, nous avons compté comme salaire les sommes versées par l'État aux particuliers.

Le partage du revenu national entre les différents facteurs de production se présente donc ainsi: ${ }^{2}$

1 Lavoisier dit: 'La consommation des Parisiens se montant à 260 millions et leurs économies à 40 millions, leur revenu atteint 300 millions': soit $Y=C+S$.

${ }^{2}$ Au lieu de retrancher. les impôts indirects et de partager le revenu national au coût des facteurs entre les facteurs de production usuels, nous avons, dans un but d'unité de calcul, considéré que les sommes payées à l'État en impôts indirects venaient en rémunération $d^{\prime} u n$ facteur de production, que nous pourrions appeler 'activité législative' (legislatorship). Il est clair que, si nous poursuivions dans cette voie, il faudrait attribuer à ce facteur l'ensemble des impôts et aux autres facteurs les revenus individuels nets des impôts directs. 


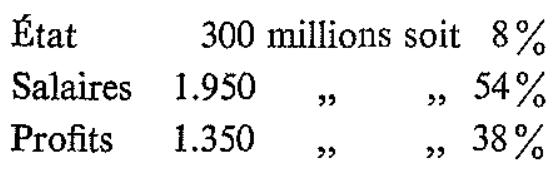

g) Comptabilité nationale. L'ensemble de ces résultats est présenté dans le tableau III. Il est inutile d'insister sur ce que cette comptabilité a de sommaire et d'inexact. Le précédent texte a montré quelles étaient les données tirées directement des sources de l'époque, quelles étaient celles qui résultaient d'une ventilation d'un chiffre entre plusieurs rubriques et quelles étaient celles qui étaient de simples hypothèses de notre part.

Nous avons porté les impôts directs comme étant entièrement payés par les non salariẻs. En fait les impôts de l'époque étaient effectivement perçus par des particuliers (fermiers généraux et autres) et reversés à l'État. Comme il est assez difficile dans les documents de l'époque de déterminer la part des sommes versées par les producteurs agricoles qui constituent des rentes, des intérêts, des profits, des impôts particuliers, survivances des droits seigneuriaux et des impôts destinés à être reversés à l'État, nous avons retiré de la valeur nette de la production agricole la consommation des cultivateurs et considéré le reste comme un profit remis aux particuliers, ces derniers acquittant ensuite l'impôt.

Comme nous avons négligé l'épargne des salariés, leur consommation est égale à leur revenu.

Sur le schéma, nous avons donné aux secteurs W et R (Salariés et Non Salariés) des surfaces proportionnelles aux nombres d'individus de ces secteurs (21 et 4). Ces valeurs sont assez hypothétiques.

Nous avons considéré le gouvernement comme un consommateur de services (fonctionnaires) produits par le secteur 'services'.

Enfin, nous n'avons pas prévu de comptes 'capital' mais nous avons distingué dans les dépenses des particuliers celles qui sont consacrées aux biens de consommation de celles qui sont consacrées aux biens d'investissement. Il en sera de même dans les autres époques. 
TABLEAU III

1788 - Comptes d'opérations

PRODUCTION (P)

\section{Agriculture (A)}

\section{Recettes}

I $\}$ Ventes aux producteurs $\left\{\begin{array}{l}300 \\ 100\end{array}\right.$

I\} Achats

$\left.\begin{array}{l}\mathrm{W} \\ \mathrm{R}\end{array}\right\} \underset{\text { teurs }}{\text { Vente aux consomma- }}$

$\left\{\begin{array}{r}1.400 \\ 350 \\ 50\end{array}\right.$

$R$ Vente de biens d'investissement

W Salaires

R Rentes et profits

$G$ Impôts indirects

Dépenses

$\left\{\begin{array}{l}100 \\ 100\end{array}\right.$

1.200

\section{Industrie (I)}

\section{Recettes}

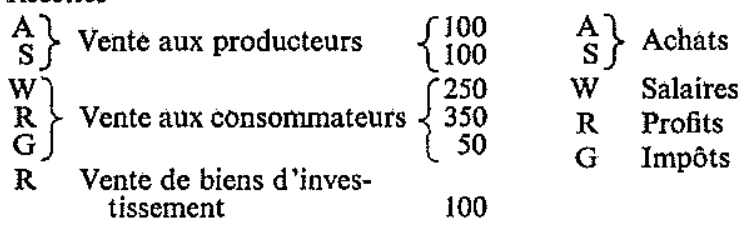

Dépenses

Services $(S)$

Recettes

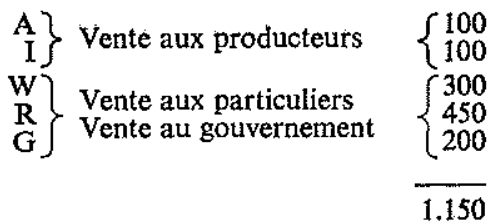

Dépenses

$$
\begin{array}{cl}
\left.\begin{array}{c}
\text { A } \\
\text { W }
\end{array}\right\} & \text { Achats } \\
\text { R } & \text { Salaires } \\
\text { G } & \text { Profits } \\
\text { Impôts }
\end{array}
$$

Production $(P)$ : Agriculture, A; Industrie, I; Services, S.

Consommation $(C)$ : Salariés, W; Non salariés, R; Gouvernement, G. 
Suite du Tableau III: 1788 - Comptes d'opérations

CONSOMMATION (C)

Recettes

$\left.\begin{array}{c}A \\ \mathbf{l} \\ S\end{array}\right\}$ Impôts indirects

R Impôts

R Emprunt

Gouvernement $(G)$

\section{Recettes}

$\left.\begin{array}{c}\text { A } \\ \text { S }\end{array}\right\}$ Salaires

Recettes

$\left.\begin{array}{c}\text { A } \\ \text { S }\end{array}\right\}$ Rentes et profits

G Intérêt de la dette

$$
\begin{aligned}
& \left\{\begin{array}{l}
100 \\
100 \\
100
\end{array}\right. \\
& 200 \\
& 100 \\
& 600
\end{aligned}
$$

A) Achat de biens

$S$ Services

$\mathrm{R}$ Dette
Depenses

$\left\{\begin{array}{l}50 \\ 50\end{array}\right.$

200

300

\section{Salariés (W)}

$$
\left\{\begin{array}{r}
1.200 \\
300 \\
450
\end{array}\right.
$$

Dépenses

$\left\{\begin{array}{r}1.400 \\ 250 \\ 300\end{array}\right.$

Non Salariés $(R)$

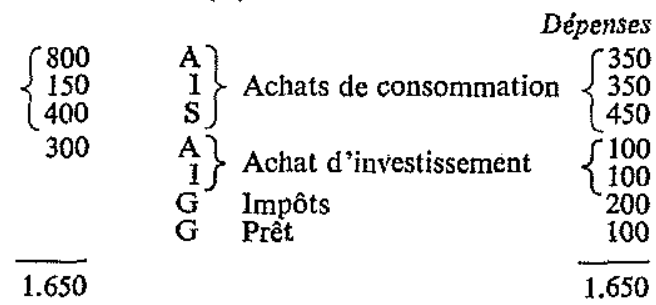

Production $(P)$ : Agriculture, A; Industrie, I; Services, S.

Consommation (C): Salariés, W; Non salariés, R; Gouyernement, G.

\section{3. $L$ 'Economie française en 1845}

a) Sources. Nos principales sources d'information ont été les enquêtes de la statistique générale de la France sur la production du royaume.

Des trois enquêtes entreprises sous le règne de Louis-Philippe et destinées à déterminer la production agricole, industrielle et artisanale de la France, seule la première put être menée à 
terme. La seconde, qui ne comprenait ni Paris, ni certaines régions du Sud Ouest peut-être assez facilement complétée, en particulier par la statistique de la Chambre de Commerce de Paris. Quant à la troisième, nous n'en avons trouvé nulle part le détail; quelques données globales se trouvent dans Moreau de Jonnès.

Les résultats de ces enquêtes ont été en effet analysés par Moreau de Jonnès dans deux ouvrages (IV) et divers articles de revue.

Le fait que ces diverses enquêtes ne soient pas exactement de la même epoque est une cause de difficulté. Nous avons tenté de donner des chiffres valables pour 1845 (date du début de l'enquête industrielle) en modifiant éventuellement les chiffres de l'enquête agricole lorsqu'il s'agissait de cultures ou d 'élevage en régression marquée. Un moyen de contrôle nous a souvent été donné par les produits de 1'agriculture servant de matière première à l'industrie dont nous trouvions une évaluation dans les deux enquêtes.

Des données partielles sur les revenus mobiliers sont fournies par Cochut (V); diverses évaluations de revenus fonciers et mobiliers sont données par Vignes (VI), Foville (VII) et divers hommes politiques à propos de projets d'imposition.

b) Agriculture. Les évaluations de la production agricole données par Moreau de Jonnès à partir des seules données de la Statistique Générale de la France varient de 4 à 8 milliards. Les différences proviennent des prix choisis (soit à la production, soit à divers stades du commerce) et des déductions faites pour se rapprocher d'un produit net. Un examen attentif de ces diverses données montre que la valeur de la production agricole vendue à l'extérieur du secteur agricole se monte à 5 milliards environ sur lesquels un milliard provient d'achats aux autres secteurs de production. Une décomposition de la valeur ajoutée est donnée dans le tableau IV.

Le contenu de la rubrique 'revenus des animaux' de la Statistique Générale de la France, est peu clair. Il s'agit le plus probablement de la somme de produits réemployés en agriculture (fumier, travail des animaux), vendus à l'extérieur (laine, lait) et de la plus-value provenant de l'accroissement du cheptel. Nous n'avons laissé subsister que ce que nous pensons représenter les deux derniers facteurs. 
TABLEAU IV

Agriculture, 1845

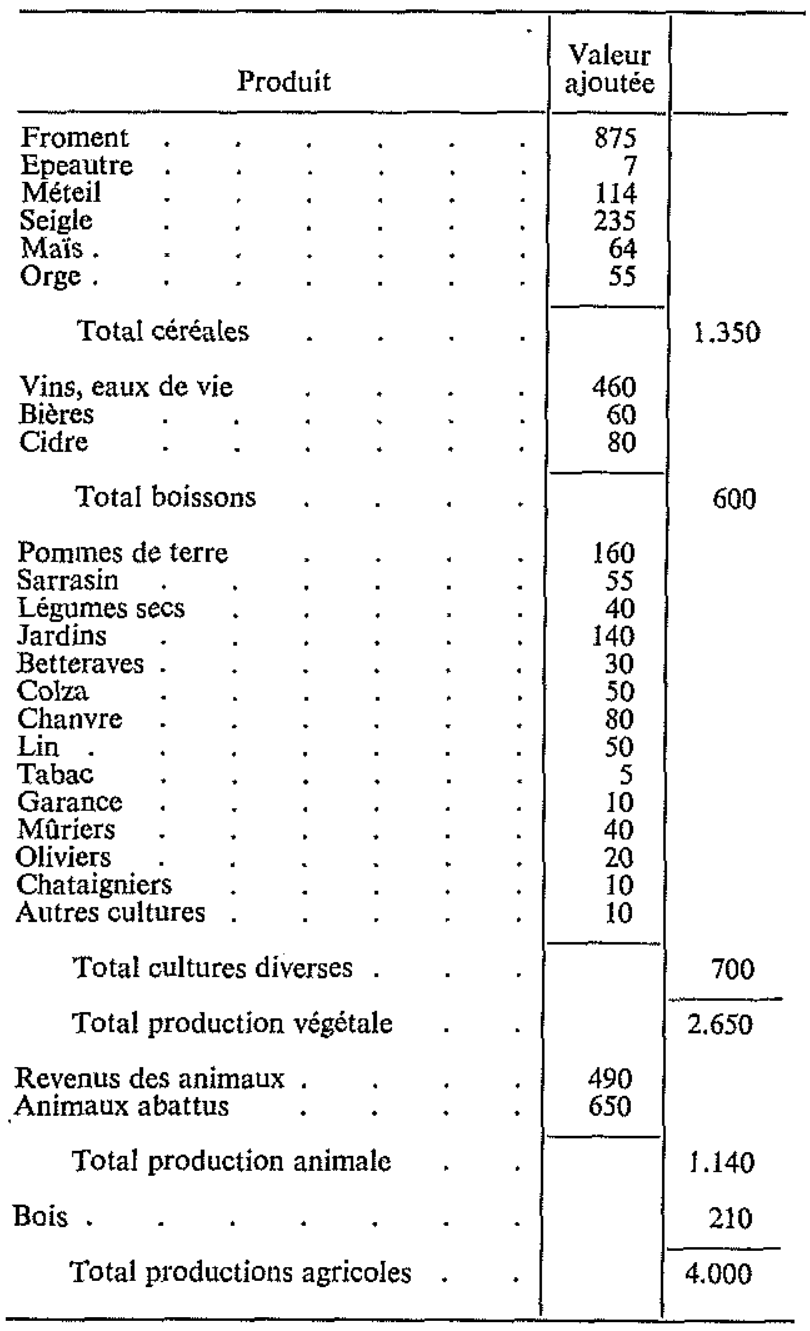

Nous avons fixé la valeur de l'amortissement à 250 millions, (l'ensemble des créances hypothécaires proprement dites se montait à 170 millions, ce qui représente une grande part de 1'amortissement des locaux), ce qui laisse 3.750 comme produit net de l'agriculture. 
Sur cette somme 200 millions sont payés en impôts indirects et 1.200 millions comme salaires d'ouvriers agricoles proprement dits (nourriture comprise); 1.300 millions constituent le revenu des propriétaires exploitants ou fermiers et le reste, soit 1.050 millions, va aux propriétaires non exploitant sous diverses formes: fermages, intérêts, etc. . . .

c) Industrie. Le tableau V donne la valeur ajoutée par l'industrie. Les matières premières importées ont une valeur de 450 millions sur lesquels 100 millions sont des droits de douane et 350 millions sont payés à l'étranger.

La valeur des machines fabriquées (100 millions environ) nous donne un élément pour estimer 1'amortissement que nous fixons, sous toutes réserves, à 350 millions, ce qui laisse 1.750 millions de produit net. ${ }^{1}$

La valeur des salaires industriels se monte à 800 millions; les impôts prélevés à la production s'élèvent à 300 millions (dont 100 de droits de douane). Il reste 650 de profits divers sur lesquels 100 millions environ sont payés aux actionnaires et 550 millions représentent du bénéfice d'entrepreneur.

d) Artisanat. Nous sommes ici réduits à des estimations plus grossières et nous fixerons à 1.300 millions le produit net de 1'artisanat, industrie du bâtiment comprise. Les salaires (y compris rémunération des petits patrons) se montent à 800 millions, les profits à 500 .

Dans notre comptabilité nationale, nous avons groupé les secteurs industriel et artisanal.

e) Commerce. Les évaluations de Cochut sur les revenus mobiliers et les différences signalées par Moreau de Jonnès entre prix à la production et prix de détail, nous servent de guide pour cette rubrique.

En y incluant les banques, nous proposons les chiffres suivants:

Valeur de la production $\quad 1.300$ millions

dont: Salaires d'employés 300

Profits 800

Impôts indirects $\quad 200$

${ }^{1}$ Dans les chiffres de la Statistique Générale, l'amortissement est inclus dans la rubrique 'Frais généraux-bénéfice'. 
TABLEAU V

Industrie, 1845

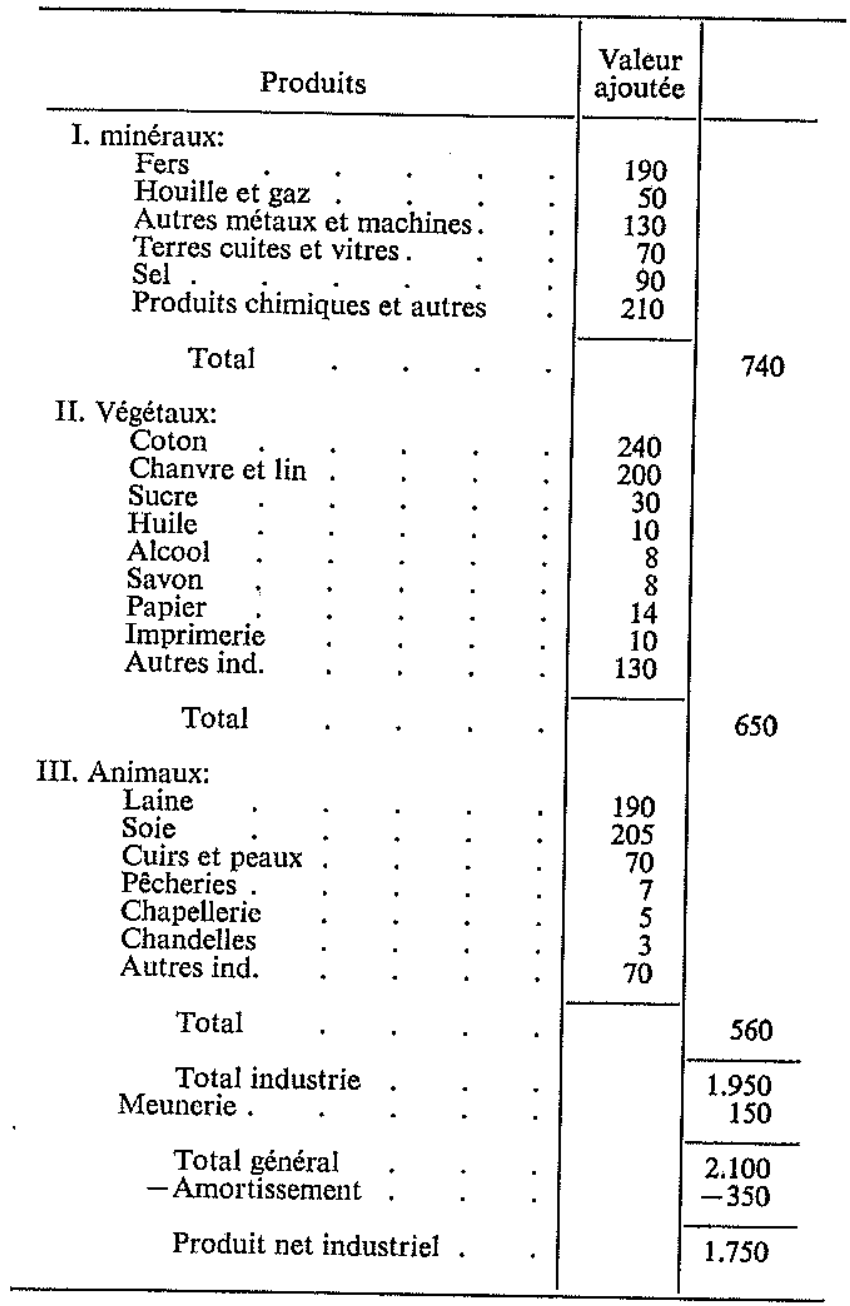

f) Professions libérales et fonctionnaires. Cochut donne 350 millions comme revenu des professions libérales proprement dites. D'autre part, une analyse simplifiée des dépenses budgétaires s'établit ainsi:

Dette

Service des Ministères 1.000

400 millions 
Dans ce dernier chiffre sont compris les frais de régie, de perception, etc. . . . Nous pensons devoir attribuer 700 millions au traitement des fonctionnaires et 300 aux achats de biens et services aux divers secteurs productifs.

g) Revenu national. Le revenu national net au prix de marché s'établit alors comme suit:

\begin{tabular}{|c|c|c|c|c|}
\hline Agriculture & \multicolumn{4}{|c|}{3.750 millions de franc } \\
\hline Industrie & 1.750 & " & $"$ & \\
\hline Artisanat & 1.300 & $"$ & , & \\
\hline Commerce & 1.300 & $"$ & " & \\
\hline Professions libérales & 350 & 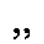 & , & \\
\hline Etat & 700 & " & " & \\
\hline R.N. net & $\overline{9.150}$ & " & ", & \\
\hline
\end{tabular}

Les divers facteurs de production y contribuent dans les proportions suivantes:

Salariés (y compris exploitants agricoles) $60 \%$

Non salariés

État (impôts indirects)

h) Utilisation du revenu national. Sous les hypothèses suivantes:

— Les salariés épargnent $3 \%$ de leur revenu (ce qui résulte des données que nous avons sur l'épargne populaire);

- Les autres consommateurs, dont le nombre est environ le huitième de celui des salariés, consomment en moyenne 3 fois plus;

- et étant donné que 1'État consomme 1.000 millions de biens et services et que la balance commerciale était excédentaire d'environ 200 millions, nous obtenons le partage suivant du revenu national:

$\begin{array}{lr}\text { Consommation privée } & 6.700 \text { millions } \\ \text { Consommation publique } & 1.000 ", \\ \text { Balance commerciale } & 200 ", \\ \text { Investissement } & 1.200 \\ \quad \text { Total } & 9.150\end{array}$

Sous ces hypothèses (peu fondées) le taux d'investissement net serait alors de $13 \%$, le taux d'investissement brut d'environ $25 \%$.

i) Comptabilité nationale. Voir tableau VI et figure 2.

j) Remarques sur ces résultats: 
1. Nous obtenons un revenu national net au coût des facteurs ( 8.400 millions) plus faible que ce que 1 'on trouve chez beaucoup d'auteurs, particulièrement ceux de l'époque. C'est que nous avons fait un effort pour obtenir un revenu national net et éviter les doubles emplois. Nous avons considéré comme 'achats à l'extérieur' des secteurs productifs, des articles alors considérés comme achats par les particuliers. Nous avons, ce faisant, considéré comme biens intermédiaires des articles considérés alors comme biens de consommation.

2. Dans notre tableau, nous avons inclus dans la rubrique salariées (W), outre les ouvriers et journaliers agricoles, les ouvriers de l'industrie et les employés du commerce, les petits exploitants agricoles, les fonctionnaires et les membres des professions libérales. Par contre nous avons inclus dans les revenus des 'non salariés' (R) ceux des petits exploitants industriels. Nous pensons ainsi avoir des secteurs assez comparables à ceux de 1788 .

3. On voit que, par rapport à 1788 , les 'services' vendent proportionnellement beaucoup plus aux salariés qu'aux non salariés. C'est qu'en 1788 la plus grande partie des salariés étaient des agriculteurs, payés pour la plus grande part en nature, logement compris. Ils payaient donc peu au commerce et la plus grande part des loyers des salariés se fondait dans les 'rentes et profits' du secteur agricole. Au contraire, en 1845, la majeure partie des salariés est composée de travailleurs libres, recevant leur salaire en argent, payant des loyers et participant à la création des bénéfices commerciaux. Nous assistons donc à un transfert de revenu des 'profits de l'agriculture' vers les 'profits des services'.

4. Une part de la croissance apparente du secteur industriel vient du transfert sur ce secteur de l'artisanat rural et de la construction rurale. Cependant la croissance de la proportion du revenu national provenant de l'industrie est manifeste. A l'intérieur du secteur industriel nous voyons que la part des industries dont la matière première est minérale a fortement augmenté.

5. Le rapport salaires sur profits semble avoir fortement augmenté dans le secteur agricole, fortement diminué dans le secteur industriel. 
TABLEAU VI

\section{5 - Comptes d'opérations}

\section{PRODUCTION (C)}

\section{Agriculture $(A)$}

\section{Recettes}

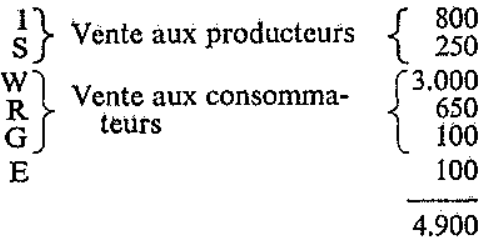
I) Achats
W Salaires
$R$ Profits
$\mathrm{G}$ Impôts indirects

\section{Industrie (I)}

\section{Recettes}

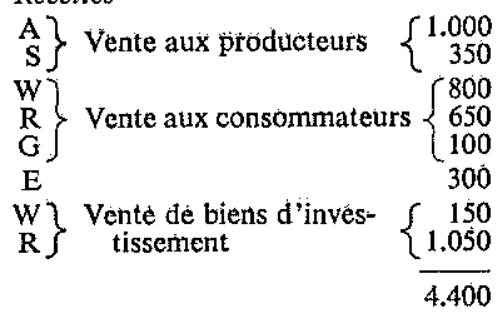
$\left.\begin{array}{l}\mathrm{A} \\ \mathrm{E}\end{array}\right\}$ Achats
W Salaire
R Profits
G Impôts

\section{Services $(S)$}

\section{Recettes}

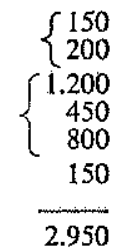

Production (P): Agriculture, A; Industrie, I; Services, S.

Consommation $(C)$ : Salariés, W; Non salariés, R; Gouvernement, G; Etranger, E. 


\section{L'INSTITUT DE SCIENCE ECONOMIQUe APPLIQUEE 83}

Suite du Tableau VI: Comptes d'Opérations, 1845

CONSOMMATION (C)

Recettes

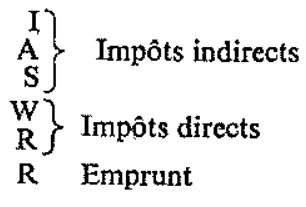

\section{Recettes}

$\left.\begin{array}{r}\text { A } \\ \text { S }\end{array}\right\}$ Salaires

\section{Gouvernement (G)}

\begin{tabular}{|c|c|c|}
\hline$\left\{\begin{array}{l}300 \\
200 \\
200\end{array}\right.$ & $\begin{array}{c}\text { A } \\
\text { I } \\
\text { S }\end{array}$ & Achats \\
\hline$\{300$ & $\mathrm{S}$ & Services (fonct.) \\
\hline$\left\{\begin{array}{r}300 \\
100\end{array}\right.$ & $\mathbf{R}$ & Intérêts de dette \\
\hline 400 & & \\
\hline
\end{tabular}

Dépenses

$\left\{\begin{array}{l}100 \\ 100 \\ 100\end{array}\right.$

700

400

$\overline{1.400}$

\section{Salariés $(W)$}

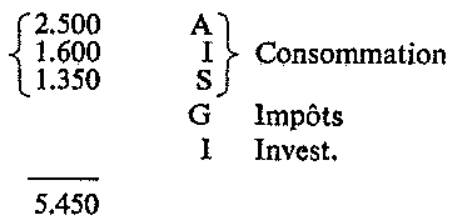

Dépenses

3.000

$\left\{\begin{array}{r}800 \\ 1.200\end{array}\right.$

300

150

$\overline{5.450}$

\section{Non Salariés $(R)$}

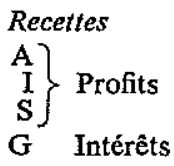

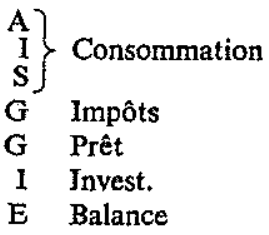

3.400
Dépenses

650

$\left\{\begin{array}{l}650 \\ 450\end{array}\right.$

300

100

1.050

200

3.400

\section{Etranger (E)}

\section{Recettes}

350

200

Dépenses

R Balance

$$
\left.\begin{array}{c}
\text { A } \\
\text { S }
\end{array}\right\} \text { Achats }
$$

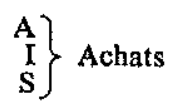

$\left\{\begin{array}{l}300 \\ 100 \\ 150\end{array}\right.$ 
4. L'Economie française en 1885

a) Sources. En dehors de données sur la population, les publications de la Statistique Générale de la France ne donnent pas de renseignements complets sur la production. Aussi nous sommes-nous servi principalement des nombreuses études faites à cette époque sur les revenus distribués, en particulier celles de Costes (VIII), Neymark (IX), de Foville (X), et Lavergne et Henry (XI) (cette dernière pour une époque plus tardive).

Nous pensons cependant qu'avec les données de la Statistique Générale de la France et celles de certains auteurs, il serait possible d'avoir une vérification en calculant le revenu national sous l'optique du produit. Seul le manque de temps nous a empêché de le faire.

Les différents auteurs divisent généralement les revenus en revenus du travail, revenus du capital et revenus mixtes. Cependant, il est clair que, particulièrement pour l'agriculture et le commerce, cette division n'est pas faite suivant les mêmes critères chez les uns et chez les autres. Nous n'avons conservé que deux catégories en nous basant sur le même principe que pour l'époque précédente.

Les résultats sont rassemblés dans le tableau VII. Si nous les discutons moins que pour les époques précédentes, ce n'est pas qu'ils nous paraissent meilleurs. C'est d'une part, qu'ils se présentent directement sous la forme que nous utilisons, et, d'autre part, par manque de temps.

Dans ce tableau:

- nous avons ignoré les transactions intérieures aux trois parties du secteur productif (Agriculture, Industrie, Services), faute de données;

- nous avons imputé aux particuliers, et non aux entreprises toutes les décisions concernant l'épargne et l'investissement;

- nous donnons une répartition des dépenses de consommation des salariés et des non salariés entre les diverses consommations. Cette répartition est très arbitraire. Nous n'en tiendrons pas compte dans nos conclusions;

- nous avons distingué des transferts les transactions qui sont des payements de produits ou rémunérations de facteurs. 
L'INSTITUT DE SCIENCE ECONOMIQUE APPLIQUEE 85

b) Revenu national net aux prix de marché. Le revenu national est ainsi constitué:

\begin{tabular}{|c|c|c|c|c|}
\hline Agriculture & \multicolumn{4}{|c|}{8.500 millions de francs } \\
\hline Industrie & 8.000 & $"$ & " & ", \\
\hline Commerce & 4.500 & " & $"$ & " \\
\hline Professions libérales & 2.000 & $"$ & " & "' \\
\hline Etat & 1.300 & $"$ & ", & ", \\
\hline R.N. net & 300 & ", & 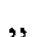 & \\
\hline
\end{tabular}

Notons que nous avons inclus dans le secteur 'professions libérales' le salaire des domestiques. Nous n'en avions pas tenu compte dans nos autres évaluations, car il était alors considéré comme un transfert et nous $n$ 'en avions aucune évaluation. Quand nous ferons des comparaisons entre ces trois époques, nous supprimerons ce revenu (1.400).

c) Utilisation du revenu national. Grâce aux travaux de Neymark nous avons des données assez précises sur l'épargne. Pendant la période 1880-1890, 1'ensemble des émissions françaises s'est monté en moyenne à 1.300 millions par an. Comme l'État émettait environ 300 millions d'emprunts par an, reste 1.000 millions pour les émissions privées. En supposant que les entreprises ne faisant pas appel au public investissaient une somme à peu près égale, nous avons fixé à 1.900 millions le montant de l'épargne privée. Nous n'avons pas tenu compte des caisses d'épargne qui investissent en général leurs fonds en emprunts d'État déjà comptés.

Faute de données précises sur l'utilisation des emprunts d'État, nous les avons considérés comme des transferts.

Pendant cette période, le portefeuille français en valeurs étrangères s'accroissait en moyenne de 500 millions par an. Le revenu des valeurs étrangères appartenant à des Français et payé en France était de 1.200 millions par an. La balance commerciale étant en déficit de 1.000 millions, nous avons balancé le compte 'Étranger' par un terme 'placements étrangers en France' de 300 millions. Nous n'avons pas, faute de données, tenu compte de la part du revenu national français allant à l'étranger par suite de tels placements antérieurs: C'est là un des défauts de logique du tableau présenté. 
Ceci étant, le revenu national est partagé de la manière suivante:

Consommation privée

Consommation publique

Investissement

Moins balance commerciale
21.300 millions de francs

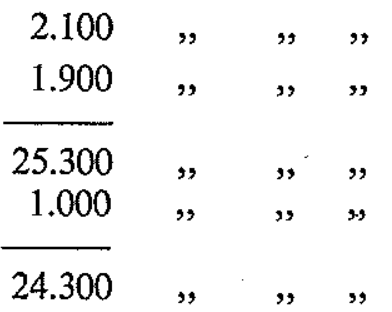

d) Contribution des différents facteurs de production. Sous les réserves déjà indiquées sur le partage des individus entre les diverses catégories, les facteurs de production concourent à la formation du revenu national dans les proportions suivantes:

$\begin{array}{lr}\text { Salariés } & 59 \% \\ \text { Non salariés } & 33 \% \\ \text { État } & 8 \%\end{array}$

TABLEAU VII

Comptabilité nationale, 1885

PRODUCTION

Agriculture $(A)$

Recettes

$\left.\begin{array}{l}\mathrm{W} \\ \mathrm{G}\end{array}\right\} \underset{\text { teurs }}{\text { Ventes aux consomma- }} \frac{\left\{\begin{array}{l}6.300 \\ 2.000 \\ 200\end{array}\right.}{\overline{8.500}}$

W Salaires

R Profits

$\mathrm{G}$ Impôts indirects

Dépenses

5.000

3.000

500

$\overline{8.500}$

Industrie (I)

Recettes

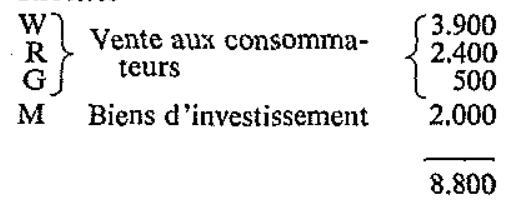

W Salaires

R Profits

G Impôts indirects

E Achats à l'étranger

Dépenses

5.000

2.500

500

800

8.800 


\section{L'INSTITUT DE SCIENCE ECONOMIQUE APPLIQUEE 87}

Suite du Tableau VII: Comptabilité nationale, 1885

Recettes

Services $(S)$ $\left\{\begin{array}{l}3.500 \\ 2.700 \\ 100 \\ 1.300\end{array}\right.$

200

$\overrightarrow{7.800}$

Dépenses

W Salaires

$R$ Profits

G Impôts indirects

4.300

2.500

1.000

7.800

\section{CONSOMMATION (C)}

Gouvernement $(G)$

\section{Recettes}

$\left.\begin{array}{c}\text { A } \\ \text { S }\end{array}\right\}$ Impôts indirects

W $\}$ Impôts directs

W $\}$ Emprunt

$\left.\begin{array}{l}\text { W } \\ R\end{array}\right\}$ Vente aux conson

\section{Recettes}

I $\}$ Salaires

G Intérêts de la dette $\left\{\begin{array}{r}500 \\ 500 \\ 1.000\end{array}\right.$

$\{300$

100

$\left\{\begin{array}{l}100 \\ 200\end{array}\right.$

$\left\{\begin{array}{l}200 \\ 100\end{array}\right.$ $\left.\begin{array}{c}\text { A } \\ \text { S }\end{array}\right\}$ Achats

$S$ (Fonct.)

$\left.\begin{array}{l}\mathrm{W} \\ \mathrm{R}\end{array}\right\}$ Intérêt de la dette
Dépenses

$\left\{\begin{array}{l}200 \\ 500 \\ 100\end{array}\right.$

1.300

$\{300$

$\{1.000$

3.400

Salariés $(W)$

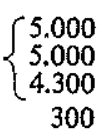

Dépenses

$$
\begin{aligned}
& \left\{\begin{array}{r}
200 \\
6.300 \\
3.900 \\
3.500 \\
100
\end{array}\right. \\
& 300 \\
& 100 \\
& 200
\end{aligned}
$$$$
G \text { Impôts }
$$$$
\text { G Prêt }
$$$$
\text { M Invest. }
$$

14.600

Non Salariés $(R)$

\section{Recettes}

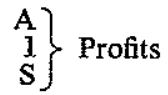

$G$ Intérêt de la dette

E Revenu de portefeuille

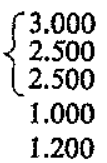
$\left.\begin{array}{c}A \\ I \\ S \\ G \\ E\end{array}\right\}$
Achats de produits de consommation
G Impôts
$G$ Prêts
$M$ Invest.
E Placement

Dépenses

2.000

2.400

2.700

100

500

200

1.700

500

$\overline{10.200}$ 
Suit du Tableau VII: Comptabilité nationale, 1885

\section{Investissements $(M)$}

Recettes

$\left.\begin{array}{l}\text { W} \\ \mathrm{R}\end{array}\right\}$ Epargne

E Placements de l'étranger

$\left\{\begin{array}{r}200 \\ 1.700\end{array}\right.$

I) Investissements

Dépenses

$\{2.000$

300

$\overline{2.200}$

$\overline{2.200}$

\section{Etranger $(E)$}

Recettes

$\left.\begin{array}{l}\text { I } \\ \mathbf{R}\end{array}\right\}$ Ventes fe produits

$\mathrm{R}$ Placements des fr. à l'étran. 500

$\left\{\begin{array}{l}800 \\ 100 \\ 100 \\ 500\end{array}\right.$

R Revenu du portefeuille fr. à l'étran.

M. Placements étran. en F.

Dépenses

$\overline{1.500}$

1.500

5. Conclusions

1) Variations dans la composition du revenu national:

Agriculture

Industrie

Commerce et services

Professions libérales

État $\frac{1788}{\%}$

58

15

18

3

6 $\frac{1845}{\%}$

41

33

14

4

8
1.200

300

Nous voyons donc une augmentation du secteur industriel et une diminution du secteur agricole, mouvements accentués de 1788 à 1845 par le passage dans nos évaluations de certaines activités du secteur agricole au secteur industriel.

Quant aux autres secteurs, je ne pense pas que nous puissions tirer de conclusions des variations enregistrées.

2) Participation des différents facteurs de productions:

Salariés

Non salariés

État

$$
1788
$$

54

$\frac{1845}{\%}$

60

38

32

8

8

$\frac{1885}{\%}$

59

33 
Nous voyons donc une remarquable stabilité de la part des impôts indirects dans le revenu national et une croíssance marquée de la part des 'salariés' de 1788 à 1845. Notons que les deux catégories 'salariés' et 'non salariés' sont des catégories comptables. Nous avons essayé d'assurer la continuité de la division, ce qui n'exclut nullement une évolution interne de nature sociologique. C'est ainsi que la catégorie 'salarié' comprend, aux diverses époques, des groupes sociaux aussi différents dans leur nature et leur évolution que le prolétariat industriel et les propriétaires agricoles exploitant.

3) Utilisation du revenu national:

Consommation privée

Consommation publique

Investissement

Balance commerciale

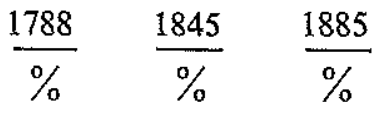

$83 \quad 74 \quad 87$

$11 \quad 11 \quad 9$

$\begin{array}{lll}6 & 13 & 8\end{array}$

$\begin{array}{lll}0 & 2 & 4\end{array}$

4) Composition de la production industrielle:

Prod. d'origine minérale $\frac{1788}{\%} \quad \frac{1845}{\%} \cdot \frac{1885}{\%}$

Prod. d'origine végétale 26

38

Prod. d'origine animale

42

33

32

29

\section{5) Remarques générales:}

Comme nous l'avons prévu, nos plus grandes difficultés au cours de cet essai d'établissement de comptabilités nationales sont venues des différences d'optique des auteurs des diverses époques, qui se traduisent par un contenu différent des notions mêmes de production et de revenu national. Un grand nombre de transactions sont passées de 1788 à 1885 de la catégorie 'transferts' dans la catégorie 'rémunération de facteurs de production'. Il nous semble même que, par rapport à 1885 , la tendance contemporaine soit un retour en arrière et que nous considérions aujourd 'hui comme transferts des transactions que les auteurs de la fin du 19ème siècle auraient classées comme 
rémunérations, particulièrement dans le secteur de l'État. Il est vrai que, aujourd 'hui comme alors, les justifications théoriques pour ce faire sont faibles.

Si nous avons vu une croissance de la part du secteur industriel aux dépens de la part du secteur agricole, le bouleversement complet des formes de production qui s'est produit au cours de l'époque étudiée n'apparaît pas dans les chiffres que nous venons de donner. C'est qu'en gros, les rapports entre les individus qu'expriment essentiellement les transactions étudiées sont restées comparables. La division des revenus en revenus provenant du travail et revenus provenant de la possession des moyens de production est valable de la suppression de l'esclavage à nos jours. Il faut aller dans le détail pour voir des variations nettes: c'est ainsi que le rapport salaire sur profit, dans le secteur industriel, est passé de 2 en 1788 à 1,4 en 1845 pour remonter à 2 en 1885, variations probablement liées à la naissance du prolétariat industriel dans la première phase et au renforcement de sa puissance et de ses moyens d'action dans la seconde.

Si nous nous posons maintenant la question - en fait préalable à l'étude des conclusions - de la confiance à accorder aux chiffres avancés, nous répondrons que nous n'avons pas pu, dans cette première ébauche, faire d'étude suffisamment poussée de la précision des observations pour pouvoir en tenir compte. Mais nous pensons que, dans une étape ultérieure, une telle étude est possible et que nous pourrons savoir quels sont ceux de nos résultats qui peuvent être significatifs. Il en serait de même pensons-nous, pour n'importe quelle période de 1830 à nos jours.

Mais nous pensons qu'une telle étude doit étre précédée d'une étude qualitative extrêmement poussée des formes de production et des rapports entre les individus dans leur activité de producteurs. Ce sont en effet ces rapports qu'expriment le cadre de notre comptabilité nationale et les rubriques que nous y incluons; l'étude des chiffres permet de remplir les colonnes de chacun de nos comptes mais ne permet pas de fixer les noms de chaque compte et de chaque rubrique. 
L'INSTITUT DE SCIENCE ECONOMIQUE APPLIQUEE 91

\section{ANNEXE \\ POPULATION}

1. Population urbaine et rurale

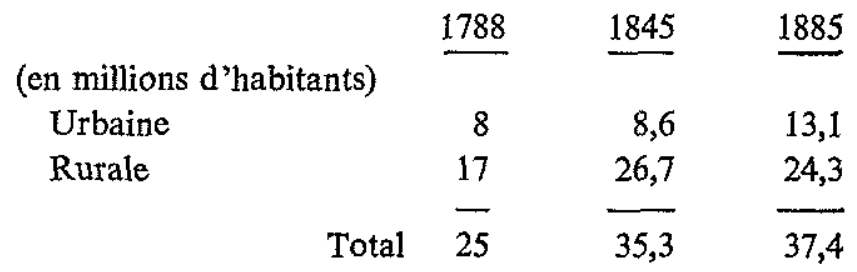

(en pourcentages)

$\begin{array}{llll}\text { Urbaine } & 32 & 24,5 & 35 \\ \text { Rurale } & 68 & 75,5 & 65\end{array}$

Les chiffres de 1788 sont donnés sous toutes réserves par Lavoisier. Les autres résultent des recensements quinquennaux.

2. Population par source de revenus

Nombre de personnes tirant leur subsistance des différents secteurs de la production.

$\begin{array}{lrrrr} & 1788 & & 1845 & \\ \text { (en pourcentage) } & & & \\ \text { Agriculture } & 75 & 62 & 49 \\ \text { Industrie } & 10 & 18 & 25 \\ \text { Commerce et services } & 8 & 6 & 12 \\ \text { Professions libérales } & 5 & 5 & 6 \\ \text { Autres } & 2 & 9 & 8\end{array}$

Ces données, particulièrement celles de 1788 , sont peu sûres et demandent à être revues et précisées.

\section{Population ouvrière}

Proportion de la population totale vivant d'un travail salarié dans les manufactures et usines. Les salariés agricoles et ceux de l'artisanat sont exclus ainsi que les employés de commerce.

$$
\begin{array}{llll}
\text { (en pourcentages) } & \frac{1788}{2} & \frac{1845}{4,5} & \frac{1885}{15}
\end{array}
$$


I. LAVOISIER.

II. (TOLOSAN).

III. BRAESCH.

IV. MOREAU DE JONNES. Statistique de l'agriculture de la France, Paris, 1848. Statistique de l'industrie de la France, Paris, 1856. Eléments de Statistique, Paris, 1856.

Statistique générale de la France en 13 volumes, publiés par la Statistique Générale de la France.

V. COCHUT.

VI. VIGNES.

VII. DE FOVILLE.

VIII. Coste.

IX. NEXMARK.
Résultats extraits d'un ouvrage intitulè: $D e$ la richesse territoriale du royaume de France, Paris, 1791. (Plusieurs fois réédité, en particulier dans la collection des principaux économistes T. XIV.)

Mémoire sur le commerce de la France et de ses colonies, Paris, 1789. (Publié sans nom d'auteur.) In- $8^{\circ}$.

Les finances et la Monnaie Révolutionnaires. Fascicule 2: Le compte rendu du Roi de 1788.
De la distribution des richesses à I'occasion de l'impôt sur le revenu, Revue des Deux-Mondes, janvier 1849.

Traité des Impóts en France, Paris, 1880, Tome II.

La France Économique, 1887 et 1889 , Paris.

Article dans le Journal de la Société de Statistique de Paris, acǔt 1890.

Bulletin de l'Institut International de Statistique, Tome XIX, Livre II, La Haye, 1912.

De 1789 à 1889, Joumal de la Société de Statistique de Paris, 1889

Article dans Le Rentier du 17 octobre 1911.

X. Lavergne et Henry. La richesse de la France, Paris, 1908. 
L'INSTITUT DE SCIENCE ECONOMIQUE APPLIQUEE 93

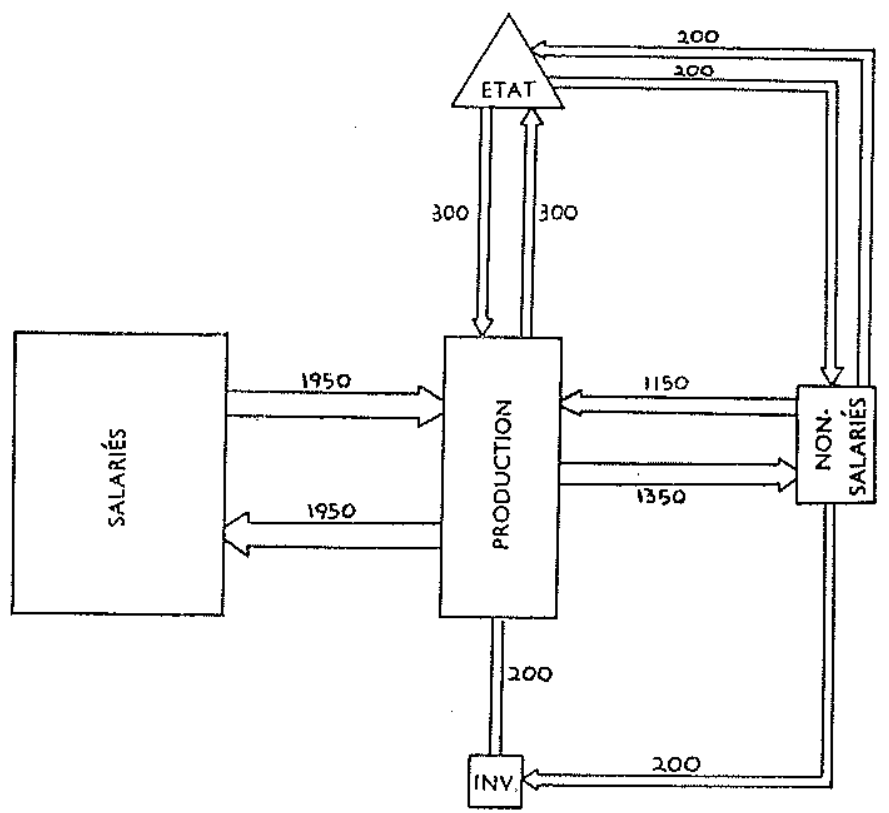

Diagramme A. La Structure de L'Economie en 1788 


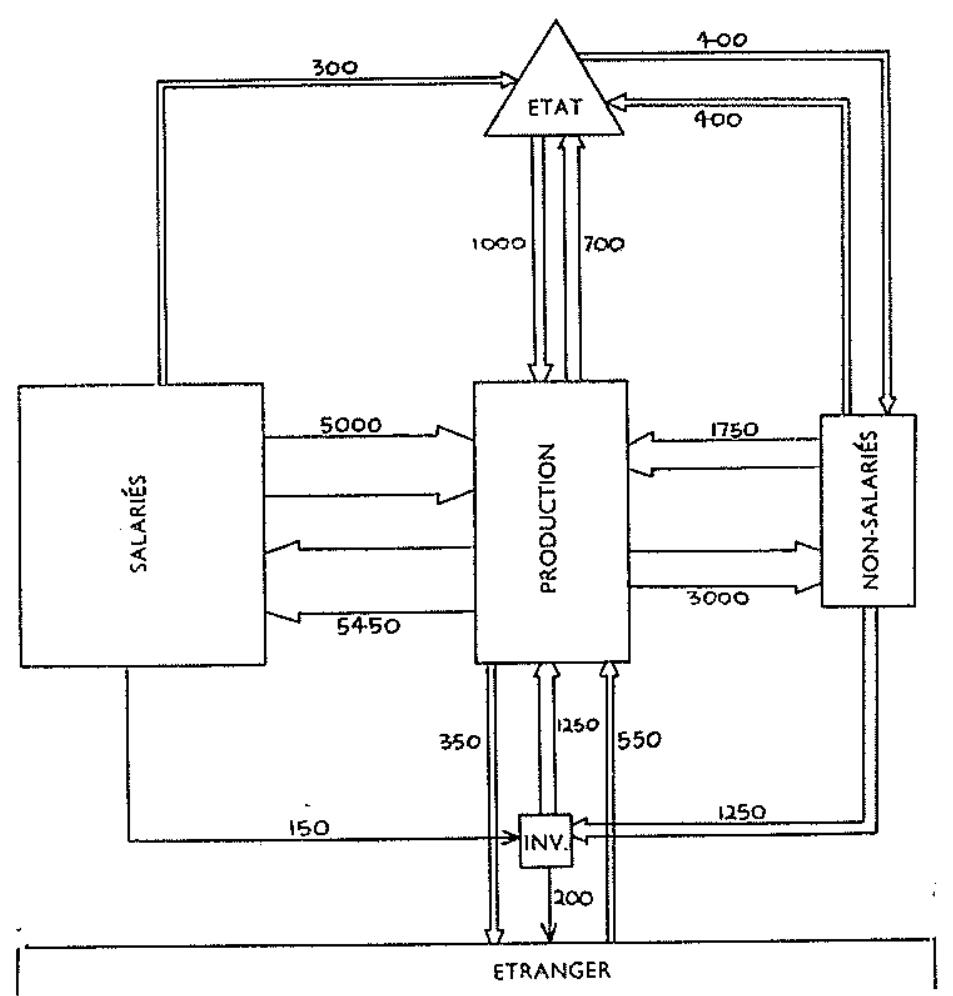

Diagramme B. La Structure de L'Economie en 1845 
L'INSTITUT DE SCIENCE ECONOMIQUE APPLIQUEE 95

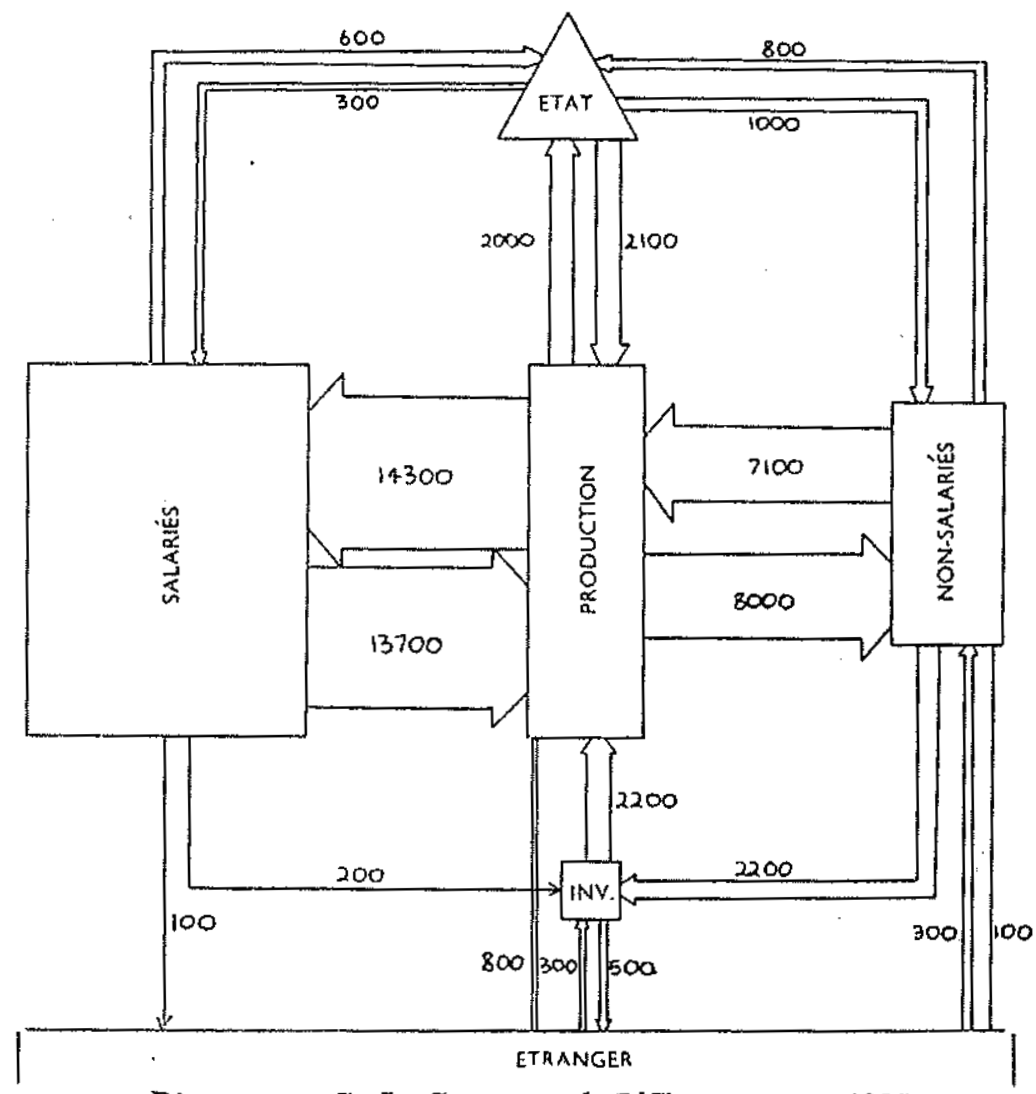

Diagramme C. La Structure de L'Economie en 1885 


\section{ENGLISH SUMMARY}

\section{THE NATIONAL INCOME OF FRANCE SINCE 1780}

1. This report is a collective work, prepared under the direction of François Perroux by Georges Guilbaud and Jacques Mayer, with the assistance of Jean Albert and Marcel Malissen. All members of the team wish to emphasize as definitely as possible that, in their opinion, the history of an economic growth cannot be told by the fluctuations of a single magnitude (whether it be national product or income; average product or income per capita; or any single index), but must be described as the changing pattern of the set of figures which constitutes the national accounts. In a few words, it is not merely a matter of aggregate quantities, but mainly a matter of economic structure, and the most important problem in such a topic is to make clear how something which we call growth has occurred, at what cost - or at the expense of whom - and to what profit or to the profit of whom.

Nevertheless, aggregate estimates are useful as an introduction to the problem. They are dealt with in the first part of our report. The second part is devoted to a draft of national accounts at three distant periods and, as far as possible, to their comparison.

2. It is often said that the statistical data on national income since the end of the eighteenth century are very abundant in France. That is true: we used more than fifty estimates of national income for the nineteenth century alone, selected amongst an even greater number. These estimates are fairly regularly scattered over the period, and their thorough examination shows that it should be possible to draw, out of them and of the computations they are made of, national accounts for a satisfactory number of periods.

But one has to be careful when reading most of the lists of aggregate data published up to now: the too frequent lack of comments prevents one from giving any significance to the figures so gathered, for all of them do not correspond to the same quantity. Moreover, some authors (including Mr. Colin Clark), who could not get to the primary sources, have contented themselves with secondary information without noticing that they were quoting twice, if not more often, the same estimates slightly altered or not even altered at all by the 
L'INSTITUT DE SCIENCE ECONOMIQUE APPLIQUEE 97 successive statisticians, economists or politicians who had made use of them.

Our effort of selection has not always been to get rid of estimates which were not likely to result from an accurate calculation (it may be useful to compare a rough opinion with a thorough computation). It has been:

(i) To put aside the estimates which, though precise, reliable and certainly useful for a later stage of our study, are not complete, that is to say cannot pretend to express any one of the usual totals admittedly representing a national income;

(ii) To discover the exact origin of the complete estimates we have come across and to eliminate the redundant ones;

(iii) To make clear, as far as possible, the character of each of these genuine estimates, as well as the character of the men who produced them;

(iv) To divide our set of estimates into two parts: on the one hand, those which belong to series established by one person and are therefore likely, within any given series, to represent the same quantity; on the other hand, all the isolated computations.

3. The paper deals first with the series: there are 35 estimates in 10 series. They are plotted on a chart in semi-logarithmic co-ordinates. They differ in magnitude, of course, for any given period, but it is remarkable that all the lines linking the points of a given series are fairly parallel, which means that the relationship between the different significant totals is roughly one of proportionality over time. Thus, it becomes possible, if we seek merely a typical rate of growth, to re-group the 35 estimates by translation to a common starting-point. It appears, then, that the rate of growth is about 1.8 per cent per year, which means 20 per cent every 10 years and 100 per cent in 39 years.

Was this rate steady or fluctuating? The line which most closely fits the 35 points is slightly fluctuating in accordance with the kondratief cycles, but that does not allow to conclude in favour of anything because its waves are entirely within the margin of error which can reasonably be expected (15 per cent more or less).

4. So far, monetary values only were taken into account. That 
is of minor importance, owing to the monetary stability which prevailed throughout the nineteenth century and even until 1914. If, however, we apply a price index to the previous figures, we find a slightly smaller rate of growth.

After 1914 it is obviously necessary to correct for the ample variations of prices which have occurred since then. It seems that the rhythm of the growth has considerably changed during this later period.

As for the growth of population, it has been very slow for the last 150 years, so that the relationship between economic and demographic growths is hardly apparent.

If it is not very surprising that the regularity of growth of the French national income, between 1780 and 1914, was roughly independent of the monetary and demographic conditions, which did not change much, it is more striking that, at least on the scale of our computations, it does not seem to have been altered by the political and social background, which was changeable, as everyone knows.

5. We now introduce the isolated estimates. The dispersion does not increase much. If, therefore, we wanted to express only the fact of the growth and to estimate a mean rate, the accuracy given by our sources would be quite sufficient. But if the point is, as we believe, to interpret the phenomenon, then the main difficulty arises from the precision required in statistical estimations: the most significant phenomena occur within a margin which, at present, is included within the margin of general uncertainty.

6 . Let us pass to the draft of national accounts for three oneyear periods dated approximately $1788,1845,1885$. In the first period the physiocratic theories were still holding very fast and the statistical apparatus was not well organized. In the second one, industrial production was in the limelight, French administration was efficient, and the Statistique Générale de la France, which had been set up for fifteen years or so, was working well. In the third period the current economic thought had changed, as well as the political and social background, and the structure of industrial production had become more and more complicated.

As a result, it happens that the national income is mainly considered from the point of view of consumption in the first 
period, from the point of view of production in the second, and from the point of view of earnings in the third.

7. On that ground the three sets of national accounts are presented as follows:

(i) The aggregate national product for 1788 and for 1845 and the aggregate national income for 1885. An attempt at breaking down these aggregate quantities into sectors of production has been made, very successfully for 1845 , satisfactorily for 1788 , but rather unsuccessfully for 1885 , since, for this last period, the net national income at market prices could not be broken down into more than five groups (agriculture, industry, trade, professional incomes, government).

(ii) An estimate of the respective shares of consumption and investment, and a tentative allocation of consumption to agriculture, industry, services.

(iii) A tentative distribution of the national income in three broad categories: government revenue, wages, profits.

(iv) Drafts of national accounts, showing receipts and outlays on the production and on the consumption sides, the sectors of production being agriculture, industry, services, the sectors of consumption being government; wage earners, non-wage earners, and the item being the particular sorts of flows linking each group of producers to each group of consumers. For 1845 and 1885 an account has been introduced for foreign trade, which is neglected in 1788 , when it was very small and its balance averaged zero.

Subject to qualifications which are expressed in the report, the following sets of figures are also given, in percentages for each of the three selected periods:

- structure of the net national product (agriculture, industry, trade and related services, professional services, government);

- structure of the next national income (wages, other personal income, government);

- structure of the national expenditure (private consumption, public consumption, net investment, balance of external trade). 
For 1788 and 1845 the structure of industrial production (from mineral, vegetable and animal raw materials) is also given.

8. The outstanding conclusions of this reconstitution of past accounts may be stated as follows:

(i) The comparison between the accounts at the three distant periods considered is rendered somewhat awkward, owing to the different approaches utilized, and, consequently, to the very content of the concepts of national income and national product;

(ii) Many transactions, quoted as transfers in the last years of the eighteenth century, came more and more to be computed as earnings during the nineteenth; it seems that this tendency culminated towards the end of the nineteenth century and has been ebbing in contemporary thought;

(iii) The three series of computations show, of course, the growth of industry relatively to agriculture, but do not reveal the complete change in the economic and social structure which has occurred during the last 150 years;

(iv) Any serious study of economic growth, if one wants it to be made in terms of reliable accounts, must be based upon a very thorough preliminary study of the forms of production and of productive relations successively involved. 